\title{
Multifunctional serine protease inhibitor-coated water-soluble gold nanoparticles as a novel targeted approach for the treatment of inflammatory skin diseases
}

David Limón, ${ }^{\not, \neq}$ María José Fábrega, ${ }^{\S, l}$ Ana C. Calpena, ${ }^{\ddagger}, \mid$ Josefa Badia, ${ }^{\S, \|, *}$ Laura Baldomà, ${ }^{\S, \|}$

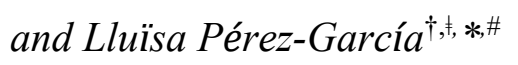

†Departament de Farmacologia, Toxicologia i Química Terapèutica, Universitat de Barcelona, Av. Joan XXIII, 27-31, 08028 Barcelona, Spain.

${ }^{\ddagger}$ Institut de Nanociència i Nanotecnologia (IN2UB), Universitat de Barcelona, Av. Joan XXIII, 27-31, 08028 Barcelona, Spain

${ }^{\S}$ Secció de Bioquímica i Biologia Molecular, Departament de Bioquímica i Fisiologia, Facultat de Farmàcia i Ciències de l'Alimentació, Universitat de Barcelona, Av. Joan XXIII, 27-31, 08028 Barcelona, Spain.

Institut de Biomedicina de la Universitat de Barcelona (IBUB); Institut de Recerca Sant Joan de Déu (IR-SJD), Barcelona, Spain.

Departament de Farmàcia, Tecnologia Farmacèutica i Fisicoquímica, Universitat de Barcelona, Av. Joan XXIII, 27-31, 08028 Barcelona, Spain. 
KEYWORDS: Serine protease inhibitors, Water soluble gold nanoparticles; Skin diseases; Drug delivery; Kallikrein; Keratinocytes; Rosacea.

ABSTRACT: The overexpression and increased activity of the serine protease Kallikrein 5 (KLK5) is characteristic of inflammatory skin diseases such as Rosacea. The use of inhibitors of this enzyme - such as 4-(2-aminoethyl)benzenesulfonyl fluoride hydrochloride (AEBSF·HCl) or the anti-human recombinant Kallikrein 5 (anti-KLK5) antibody - in the treatment of the disease has been limited due to their low bioavailability, for which their immobilization in drug delivery agents can contribute to making serine protease inhibitors clinically useful. In this work, we synthesized gold nanoparticles (GNP) coated with a mixture of hydroxyl- and carboxyl terminated thiolates (GNP.OH/COOH), whose carboxyl groups were used to further functionalize the nanoparticles with the serine protease inhibitor AEBSF $\cdot \mathrm{HCl}$ either electrostatically or covalently (GNP.COOH AEBSF and GNP.AEBSF, respectively), or with the anti-KLK5 antibody (GNP.antiKLK5). The synthesized and functionalized GNP were highly water soluble, and they were extensively characterized using UV-VIS absorption spectroscopy, Transmission Electron Microscopy (TEM), Dynamic Light Scattering (DLS) and Thermogravimetric Analysis (TGA). GNP.OH/COOH and their subsequent functionalizations effectively inhibited KLK5 in vitro. Internalization of fluorophore-coated GNP.OH/COOH in human keratinocytes (HaCaT cells) was proven using confocal fluorescence microscopy. Cell viability assays revealed that the cytotoxicity of free AEBSF is importantly decreased when it is incorporated in the nanoparticles, either ionically (GNP.COOH AEBSF), but most importantly, covalently (GNP.AEBSF). The functionalized nanoparticles GNP.AEBSF and GNP.antiKLK5 inhibited intracellular KLK5 activity in $\mathrm{HaCaT}$ cells and diminished secretion of IL-8 under inflammatory conditions triggered by TLR-2 ligands. This study points to the great potential of these GNP as a new intracellular 
delivery strategy for both small drugs and antibodies in the treatment of skin diseases such as Rosacea.

\section{Introduction}

Rosacea is a chronic, inflammatory skin disease characterized by erythema, telangiectasia and papulopustules. ${ }^{1,2}$ Its etiology is not completely known yet and pharmaceutical products on the market commonly contain drugs that only act upon the medical symptoms. ${ }^{3-5}$

As the first line of defense against pathogens, keratinocytes express the Toll-like receptor TLR2 on their cell surface and in endosomal compartments, which can be activated by external agents. ${ }^{6,7}$ In Rosacea patients, the overexpression of TLR-2 enhances the sensitivity to external agents, increasing the expression and activity of the serine protease Kallikrein-5 (KLK5) ${ }^{8,9}$ In addition, the proteolytic activity of KLK5 is altered in this disease, producing pro-inflammatory cathelicidin peptides, which in turn leads to an increased IL-8 secretion. ${ }^{1,6-9}$ An overall biochemical pathway was drawn according to reports, ${ }^{1,8-11}$ and is represented in Figure 1. Enzymatic activity of KLK5 is regulated by endogenous serine protease inhibitors (SERPINS); ${ }^{12}$ however, in Rosacea patients, endogenous SERPINS seem not to have enough efficacy. The use of serine protease inhibitors as an attempt to treat Rosacea has been reported a few times, ${ }^{13-15}$ but the low bioavailability of some of them, such as AEBSF $\cdot \mathrm{HCl}$ or the anti-KLK5 antibody, makes their conventional administration still limited. Therefore, the use of carriers in the administration of enzymatic inhibitors could help solving the problem for the regulation of KLK5 activity, helping to consolidate this new therapeutic strategy in Rosacea.

Gold nanoparticles (GNP) are becoming a main interest as a platform for biomedical applications such as sensing and therapy, for their multiple advantages such as chemical stability, low toxicity, 
high extinction coefficient, and capability for varying the size and the external functionalizations according to the reaction conditions. ${ }^{16-18}$ According to the Enhanced Permeability and Retention Effect (EPR effect), the nanometric size of GNP promotes their selective extravasation from blood vessels to the inflamed tissues, where the permeability of vessels is higher, ${ }^{16,19,20}$ as is the case of the skin in patients with Rosacea. ${ }^{2,4,5}$ Therefore, GNP functionalized with the appropriate drugs can provide a passive targeting to the skin in Rosacea patients, decreasing the dose needed to get an effective treatment, as compared to the administration of the drug alone.

Also, the functionalization with chemical entities which are capable of recognizing specific biomolecules, such as the case of antibodies, promotes retention of the particles around the tissues where their recognized antigens are located, providing an active targeting. For instance, the formation of a complex of GNP with an anti-KLK5 antibody could increase the concentration of such complex at the skin. Moreover, it is known that the size of GNP, as well as their coating agents, greatly influence their internalization in cells. For instance, GNP coated with PEG molecules can be more easily internalized in cells by increasing the length of the PEG chain and by decreasing the particle size. ${ }^{21}$

For these reasons, in this work PEG-coated gold nanoparticles were synthesized and functionalized with the well-known serine protease inhibitor AEBSF· $\mathrm{HCl}$ (covalently or electrostatically) or with the anti-KLK5 antibody. All these nanoparticles were extensively characterized, and their ability to be internalized in keratinocytes and to inhibit intracellular KLK5 and IL-8 secretion was assessed. The results suggest the potential of GNP to become an efficient new therapeutic approach for the efficient delivery of drugs for Rosacea treatment. 


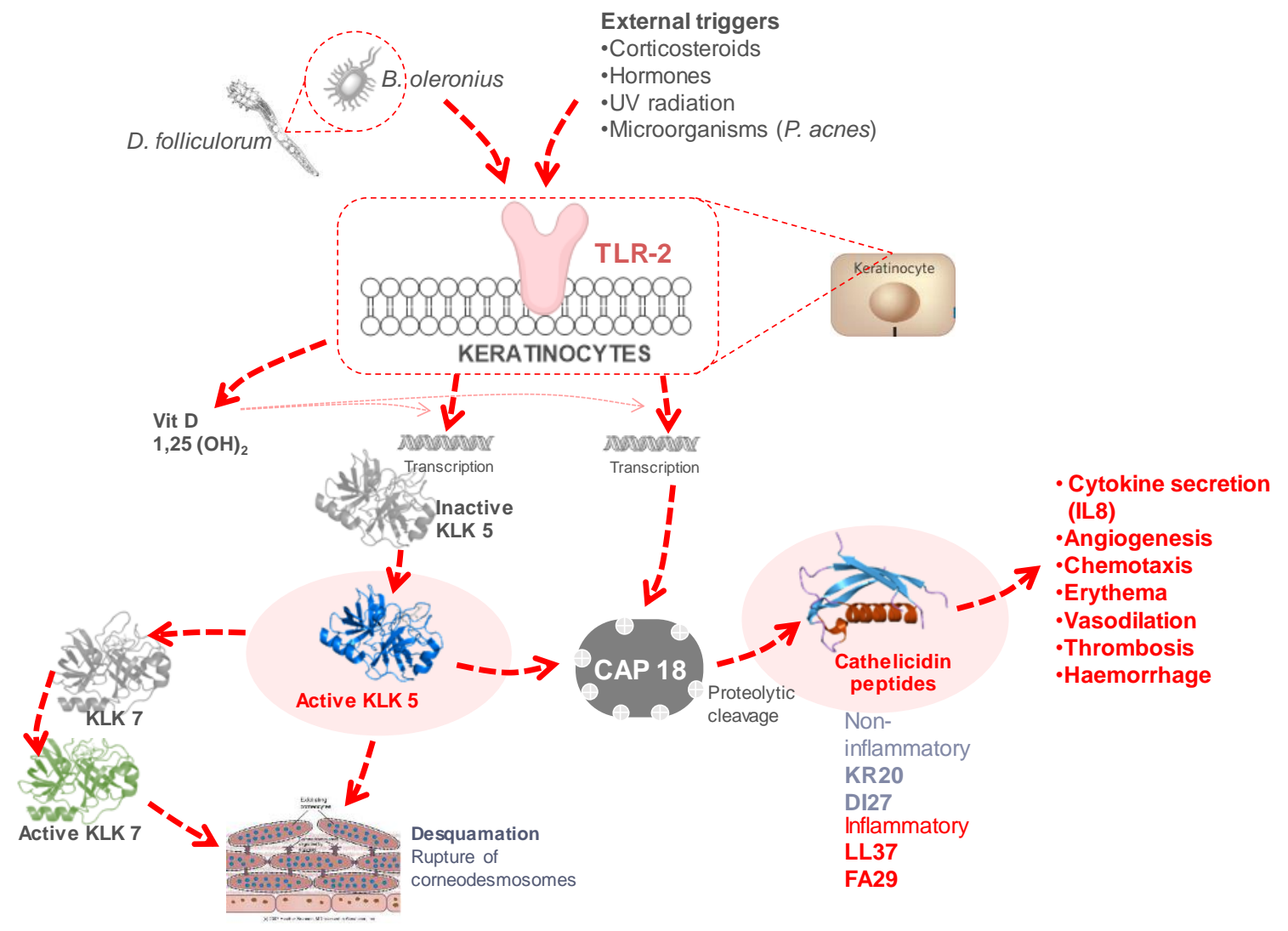

Figure 1. Biochemical pathway of Rosacea. The activation of TLR-2 receptors by external agents promotes by different routes the activation of KLK5. KLK5 activity is augmented and altered in Rosacea patients, leading to proinflammatory cathelicidins that orchestrate the inflammatory response. Adapted from ${ }^{1,8-11}$

\section{Results and discussion}

\section{Synthesis and characterization of GNP.OH and GNP.OH/COOH gold nanoparticles}

With the aim of functionalizing gold nanoparticles with different serine protease inhibitors, our initial design was the synthesis of gold nanoparticles coated with one kind of thiolated-PEG molecules that include a terminal carboxyl group (PEG.COOH). The synthesized particles, GNP.COOH, showed an SPR band near $\lambda=515 \mathrm{~nm}$ using UV-VIS absorption spectroscopy. These particles remain in solution after centrifugation for $30 \mathrm{~min}$, for which they can be considered 
highly-water soluble. However, further functionalization with serine protease inhibitors yielded nanoparticles with low water solubility, making them unsuitable for biological applications, and which were no longer used in further experiments.

In order to obtain highly-water soluble particles, we considered using mixed coatings by including as well the thiolated PEG ligand with terminal hydroxyl groups (PEG.OH) as a water dispersing promoter. Thus, we synthesized nanoparticles GNP.OH/COOH, comprised of a gold core coated with a mixture of two different types of thiolated-PEG molecules: one with a hydroxyl terminal group (PEG.OH), which remains unreacted and provides water solubility, and a second one with a carboxyl terminal group (PEG.COOH), which can be further functionalized with serine protease inhibitors. Also, as control particles, hydroxyl-functionalized nanoparticles (GNP.OH) were synthesized, comprised of a gold core coated with only one kind thiolated-PEG molecules (PEG.OH) with a terminal hydroxyl group. A schematic representation of these nanoparticles is shown in Figure 2.

Both GNP.OH and GNP.OH/COOH also show an SPR band near $\lambda=515 \mathrm{~nm}$ as observed by UV-VIS absorption spectroscopy (See Supporting Information, Figure S 1). Both GNP.OH and GNP.OH/COOH also remain in solution after centrifugation for $30 \mathrm{~min}$, for which they can be considered highly-water soluble as well. For this reason, centrifugation-dissolution cycles could not be used for purification of GNP.OH or GNP.OH/COOH. Instead, other methods were used, such as dialysis or filter tubes for centrifugation. GNP were preserved in a stable solution at room temperature for at least one year with no apparent sedimentation or aggregation.

As estimated by UV-VIS absorption spectroscopy, ${ }^{22}$ the GNP have an average diameter $\leq 3 \mathrm{~nm}$, with an extinction coefficient of $1.49 \times 10^{6} \mathrm{~L} \mathrm{~mol}^{-1} \mathrm{~cm}^{-1}$ in both cases. Therefore, by considering the concentration of GNP in samples and the final volume of reaction, the number of thiols per 
nanoparticle was calculated. For instance, GNP.OH was estimated to be covered by 232 PEG.OH molecules per nanoparticle, and GNP.OH/COOH covered by 121 total thiol molecules (PEG.OH + PEG.COOH) per nanoparticle, with a proportion of PEG.COOH to PEG.OH of 1:1.3.

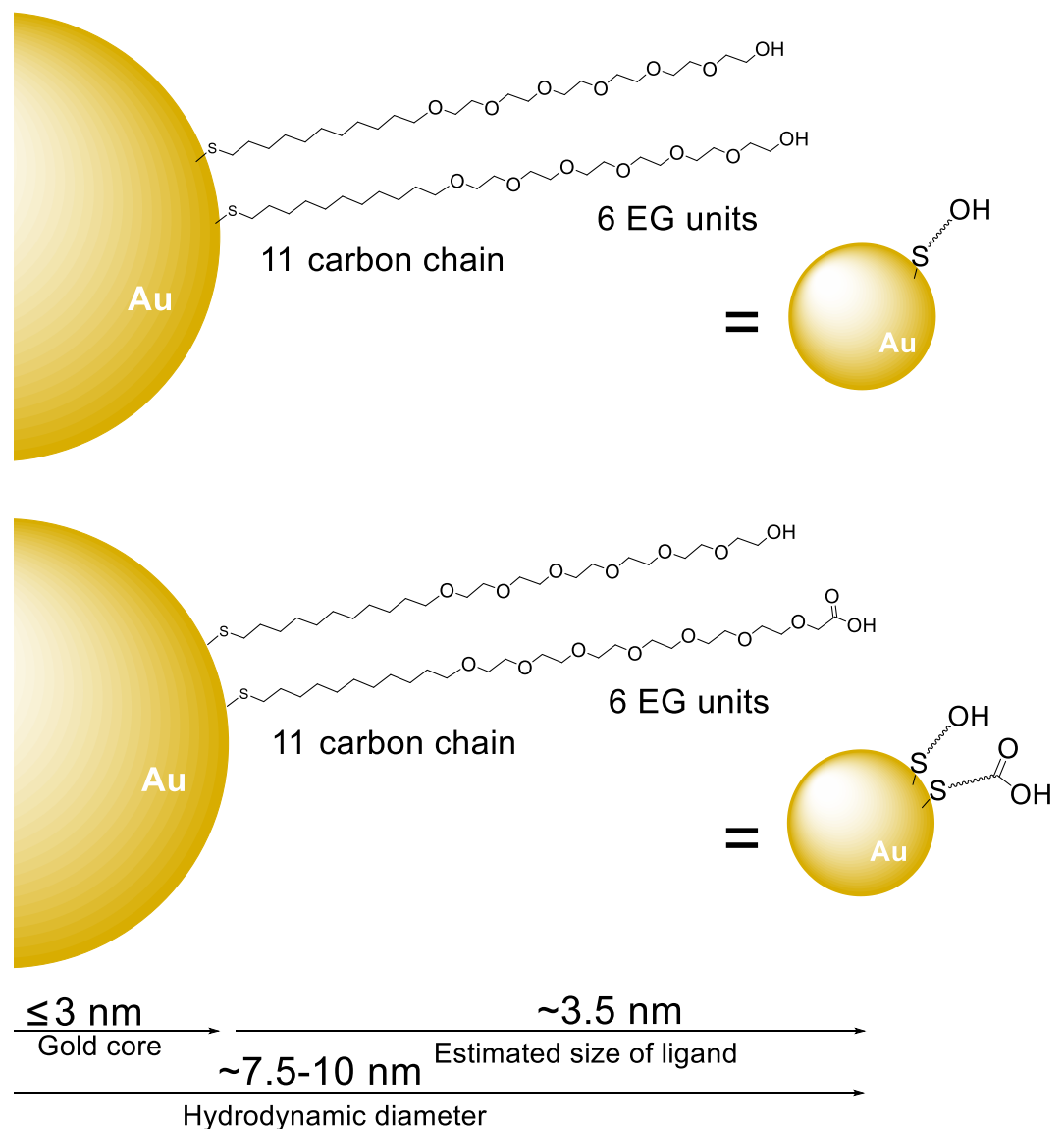

Figure 2. Schematic representation of hydroxyl functionalized gold nanoparticles (GNP.OH) as control (top), and mixed hydroxyl/carboxyl functionalized gold nanoparticles (GNP.OH/COOH) for further functionalization (bottom), in aqueous solution.

\section{Functionalization of gold nanoparticles}


GNP.OH/COOH were functionalized, using the carboxyl group from the particles surface, with different chemical entities such as the serine protease inhibitor AEBSF·HCl, linked both electrostatically (GNP.COOH AEBSF) (Figure 3, A) and covalently (GNP.AEBSF) (Figure 3, B), and the anti-KLK5 antibody covalently (GNP.antiKLK5) (Figure 3, C). All the different functionalized particles were highly water soluble, as they keep stable in solution after centrifugation for $30 \mathrm{~min}$.

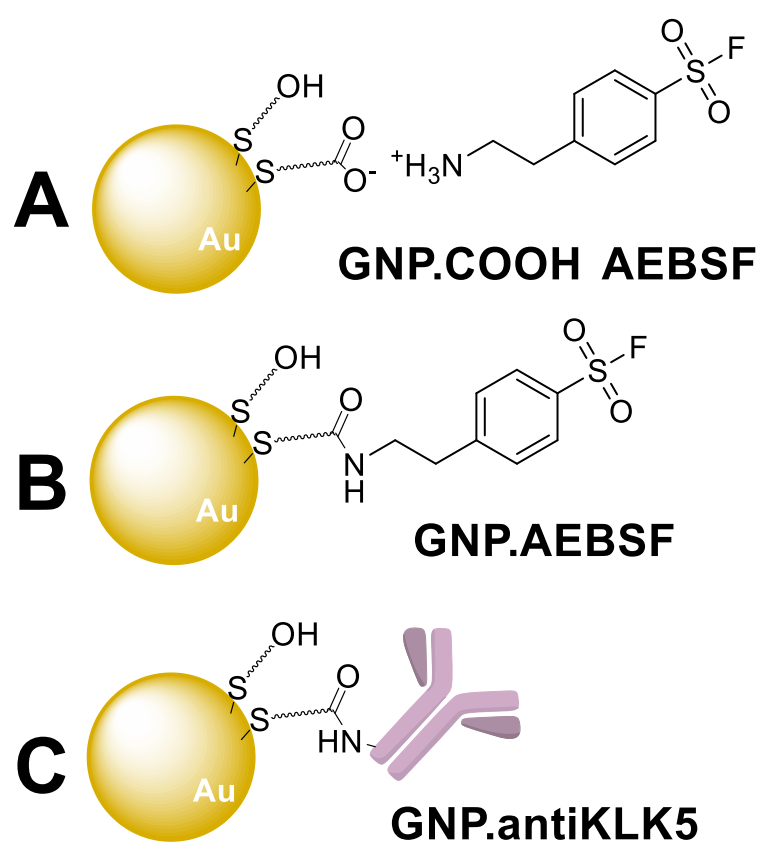

Figure 3. Ionic and covalent functionalizations of GNP.OH/COOH. A) Ionic immobilization of AEBSF (GNP.COOH AEBSF). B) Covalent immobilization of AEBSF (GNP.AEBSF). C) Covalent immobilization of anti-KLK5 antibody (GNP.antiKLK5).

In the case of GNP.antiKLK5, the resulting functionalized particles are of great importance as they could play three important roles at the same time in the treatment of Rosacea. The first role is the passive targeting, achieved by the nanometric size of the platform, permitting a much higher extravasation in inflamed tissues such as the skin in Rosacea patients, than in healthy skin. The 
second role is the active targeting, which is provided by the antibody. ${ }^{18,23-25}$ As the anti-KLK5 antibody has a high affinity for KLK5 enzyme, their specific interaction could maximize the retention of the GNP at the tissues with higher concentration of KLK5, which is the case of the epidermis, especially in patients with Rosacea ${ }^{1,9,26}$. Finally, a third role is believed to be the therapeutic potential of the the antiKLK5 itself, as it could possibly have a KLK5 inhibitory activity.

\section{Characterization of optimum GNP}

\section{UV-VIS absorption spectroscopy}

All functionalized GNP (GNP.COOH AEBSF, GNP.AEBSF and GNP.antiKLK5) were characterized using UV-VIS absorption spectroscopy (See Supporting Information, Figure S 1). They all show the characteristic SPR band at $c a . \lambda=520 \mathrm{~nm}$ as well as bands corresponding to the serine protease inhibitors they contain.). Results suggest a successful functionalization in all cases, with no evidence of aggregation.

\section{Microscopy}

The size, morphology and polydispersity of GNP were confirmed by Transmission Electron Microscopy (TEM) (Figure 4), in GNP.OH (A) and GNP.OH/COOH (B) samples. GNP.AEBSF (C), GNP.antiKLK5 (D) and GNP.COOH AEBSF (E) were also observed to prove that functionalization does not induce particle aggregation. 

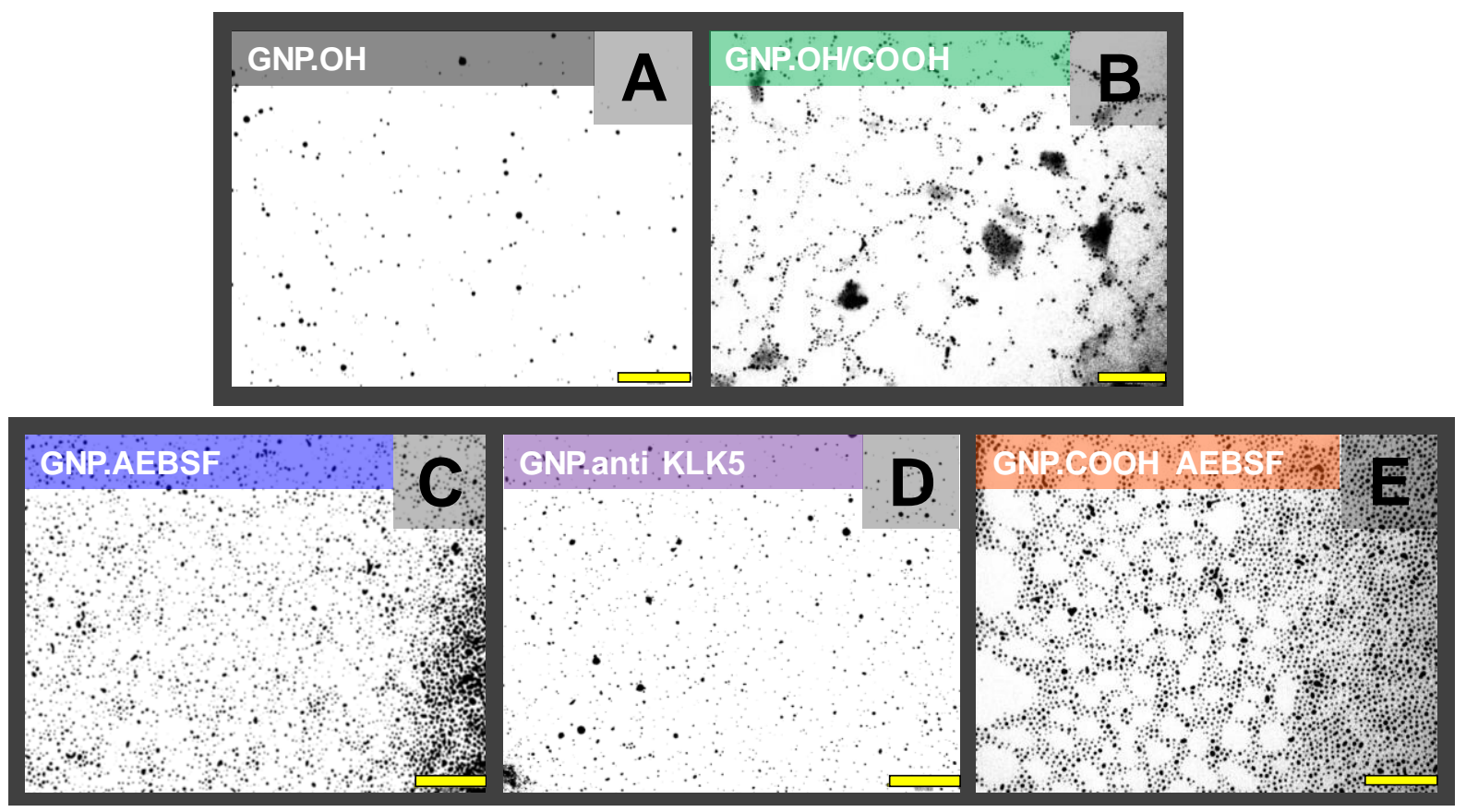

Figure 4. TEM images of different samples of gold nanoparticles. A) GNP.OH. B) GNP.OH/COOH. C) GNP.AEBSF. D) GNP.antiKLK5. E) GNP.COOH AEBSF. Scale bar represents $100 \mathrm{~nm}$.

For all samples observed, the size found for the majority of GNP was $1.5 \pm 0.7 \mathrm{~nm}$, which also was the smallest size that could be detected due to the equipment resolution; the size distribution did not follow a typical Gaussian curve, but instead, they followed a right-tailed Fisher distribution (Figure 5). Histograms show that the different GNP are monodispersed, as more than $40 \%$ of the counts have a diameter around $1.5 \mathrm{~nm}$. However, the polydispersity of GNP.OH seems to be slightly higher than the rest, as the percentage of particles above $4 \mathrm{~nm}$ in diameter is $25 \%$, while in the rest of the samples, the percentage above $4 \mathrm{~nm}$ is less than $15 \%$. This higher polydispersity can readily be observed in the plot as the slope of the curve is lower for GNP.OH than for the rest of the particles. Size distribution plots from the different GNP shown as both total counts and relative count (\%) can be seen in Supporting Information (Figure S 2). 


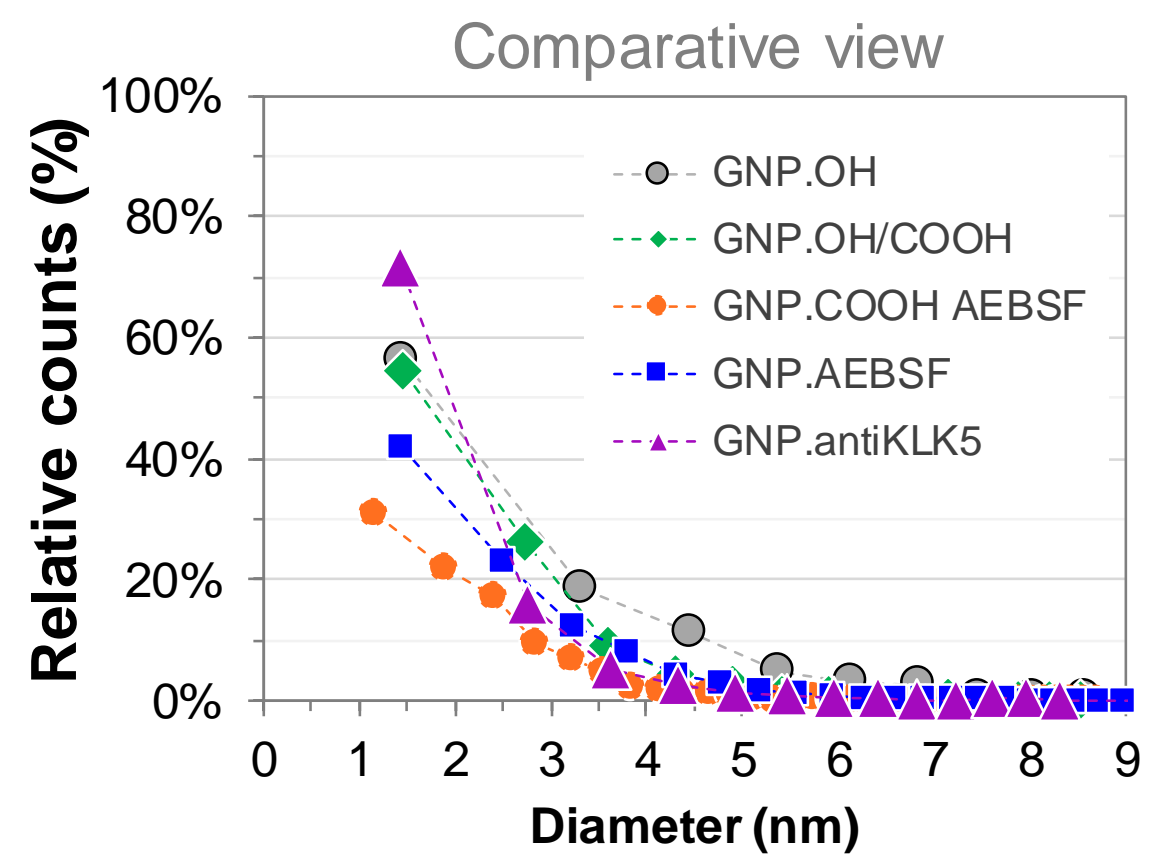

Figure 5. Size distribution of samples of gold nanoparticles GNP.OH (n=199), GNP.OH/COOH $(\mathrm{n}=583)$, GNP.AEBSF $(\mathrm{n}=2809)$, GNP.antiKLK5 $(\mathrm{n}=811)$, and GNP.COOH AEBSF $(\mathrm{n}=1778)$, expressed as a comparative view in relative counts $(\%)$.

\section{LDI-TOF mass spectrometry}

The successful covalent functionalization was proven by Laser Desorption Ionization - Time of Flight Mass Spectrometry (LDI-TOF MS), by analyzing both GNP.COOH AEBSF and GNP.AEBSF in negative mode. For GNP.AEBSF, peaks observed at $\mathrm{m} / \mathrm{z} 551$ and 566, correspond to the AEBSF molecule covalently bonded through an amide linker to the polyethylene glycol spacer, fragments including six ethylene glycol units and either a two- or three-carbon chain respectively. These results show the effectiveness of the strategy for covalent functionalization. For GNP.COOH AEBSF, peaks observed at m/z 551 and 200 and 159 correspond to fragments of AEBSF molecule, while peaks observed at m/z 177 and 103 correspond to carboxyl-terminated 
ethylene glycol fragments. As expected, no peaks indicating a covalent functionalization of AEBSF were found (See Supporting Information, Figure S 3 and Figure S 4).

\section{Thermogravimetric analysis (TGA)}

Thermogravimetric analyses were performed with GNP.OH/COOH, GNP.AEBSF, GNP.antiKLK5, GNP.COOH AEBSF and AEBSF.HCl in solution, observing mass degradations corresponding to the thiol/drug/antibody with which they are functionalized, and confirming the successful functionalization. The sample containing only the drug AEBSF $\cdot \mathrm{HCl}$ in solution shows a mass loss between $200-400{ }^{\circ} \mathrm{C}$, corresponding to the drug degradation. In the case of GNP.AEBSF, significant mass losses were observed also between $200-400{ }^{\circ} \mathrm{C}$, corresponding to big amounts of drug in the sample. In the case of GNP.antiKLK5, the mass loss in this range of temperatures was much smaller, suggesting that the percentage of surface functionalized on these particles is much less than on those small-drug-functionalized particles (See Supporting Information, Figure S 5 -Figure S 9). The sample containing GNP.COOH AEBSF also shows a mass loss around $300{ }^{\circ} \mathrm{C}$, corresponding to the drug. In this case, the biggest mass loss observed around $100{ }^{\circ} \mathrm{C}$ corresponds to high amounts of water, indicating the sample was not as dry as the rest of the particle samples. Therefore, the smaller mass loss at $300{ }^{\circ} \mathrm{C}$ as compared to GNP.AEBSF does not mean a smaller proportion of drug in the sample, but only a higher water content. Thiolated-PEG molecules were not completely degraded at the temperatures reached (950 ${ }^{\circ} \mathrm{C}$ ), as seen by the lack of a mass stabilization of the sample, for which the total mass of them cannot be known.

\section{Dynamic Light Scattering (DLS)}

GNP.OH/COOH, GNP.COOH AEBSF, GNP.AEBSF and GNP.antiKLK5 nanoparticles were analyzed by DLS to assess their hydrodynamic diameters, their polydispersity, and whether 
they are aggregated or monodisperse in solution. The diameters observed are higher than the gold core diameters observed by TEM, as hydrodynamic diameter not only considers the core but also reflects the chemical functionalization. Hydrodynamic diameters observed are quite similar in all particles under study. GNP.OH/COOH showed the highest diameter average as also the highest dispersity $(12.9 \pm 2.7 \mathrm{~nm})$, suggesting these particles are aggregated in solution. The electrostatically functionalized particles GNP.COOH AEBSF showed the smallest hydrodynamic diameter $(7.4 \pm 1.2 \mathrm{~nm}$ ), while particles covalently functionalized with AEBSF (GNP.AEBSF) or with antibody (GNP.antiKLK5) showed an increase in their diameter $(8.2 \pm 2.3 \mathrm{~nm}$ and $11.7 \pm 2.1$ $\mathrm{nm}$ respectively). The sizes observed in the different samples can only be used as an estimation of the size and polydispersity of the particles, in accordance with the sensibility of the technique (See Supporting Information, Table S 1).

Based on the results of the different techniques concerning particle sizes, a schematic representation of the nanoparticles, the different functionalizations, and their sizes is shown in the Supporting Information (Figure S 10).

\section{In vitro inhibition of KLK5}

As a first step, in vitro enzymatic assays were performed to test whether the different kinds of nanoparticles functionalized with serine protease inhibitors can inhibit KLK5 activity. Experiments were performed following the protocol described by the supplier. ${ }^{27}$ For activity assays, KLK5 was incubated with a specific fluorogenic substrate in the absence or presence of the different functionalized nanoparticles (GNP.COOH AEBSF, GNP.AEBSF, GNP.antiKLK5), as well as with the free drug AEBSF· $\mathrm{HCl}$ or the anti-KLK5 antibody for comparison. GNP.OH and GNP.OH/COOH were used as controls. In Figure 6, the percentage of inhibition calculated for each type of drug/particles was plotted against the concentration of 
functional groups responsible for such inhibition ("active groups"). In GNP.OH/COOH, the term "active groups" refers to the concentration of PEG.COOH molecules. Likewise, it refers to those PEG.COOH molecules after functionalization in case of GNP.COOH AEBSF, GNP.AEBSF, and GNP.antiKLK5. Moreover, it represents the concentration of drug in case of GNP.COOH AEBSF, GNP.AEBSF and free AEBSF·HCl. As GNP.OH is only coated with one type of thiolate with hydroxyl terminated groups, concentration was adjusted to correctly represent an equivalent concentration of total thiolates than in GNP.OH/COOH taking in account the proportion of PEG.OH to PEG.COOH ligands. This adjustment was also taken into account in the rest of experiments. In case of GNP.antiKLK5 and anti-KLK5 in solution, the concentration of antiKLK5 antibody is also shown in the axis. A broad range of concentrations was chosen in order to determine whether the inhibition follows a certain model. The best fitting model was obtained for each kind of particles/drug/antibody and the $\mathrm{IC}_{50}$ values (the concentration at which $50 \%$ of enzymatic activity is obtained) were estimated. However, it is important to note that these in vitro conditions are expected to be different than those inside the cells, including the concentration of KLK5 enzyme; for which $\mathrm{IC}_{50}$ values are not expected to be the same in cells and are calculated only for comparison purposes. 


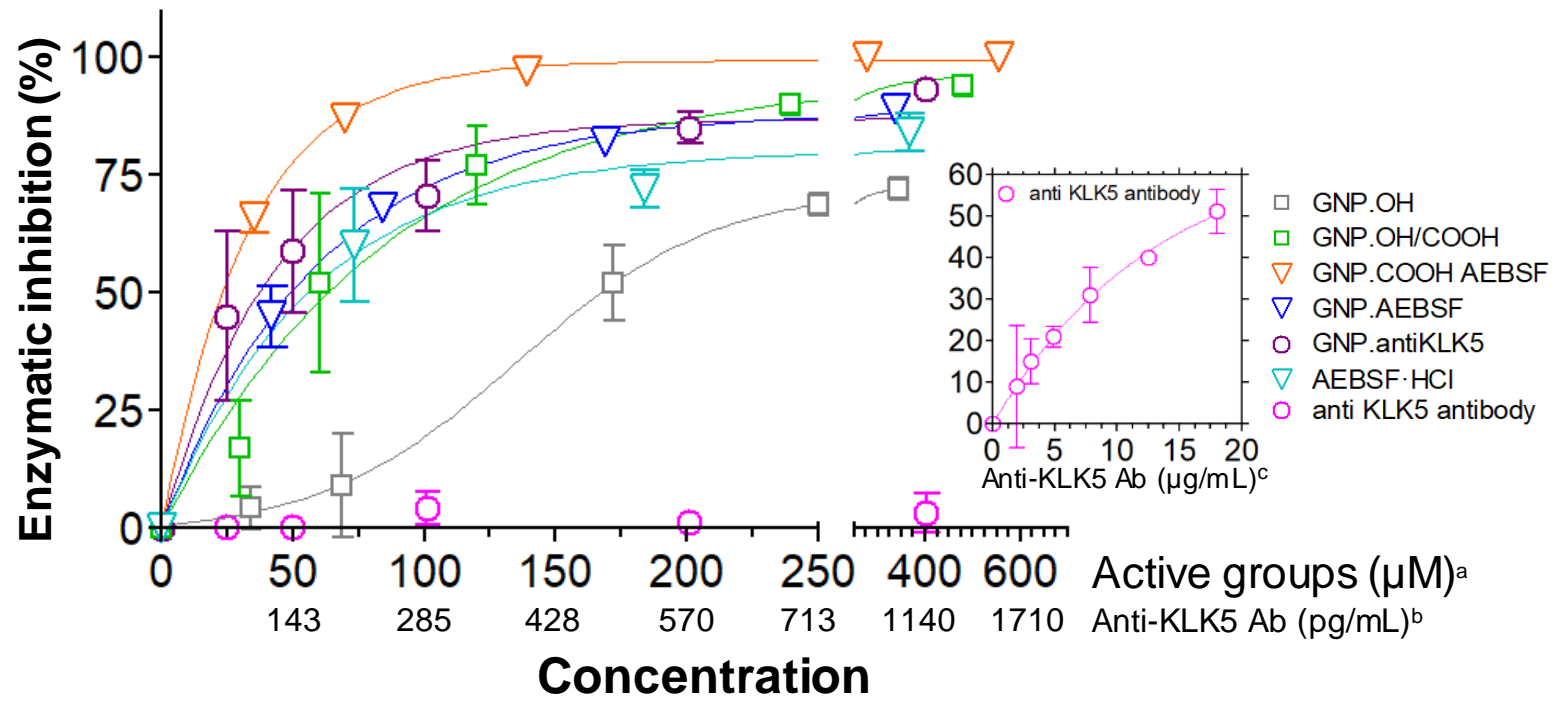

Figure 6. In-vitro enzymatic inhibition of KLK5 in presence of different gold nanoparticles at

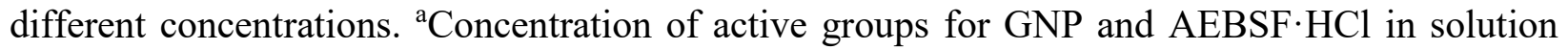
$(\mu \mathrm{M}) .{ }^{\mathrm{b}}$ Concentration of anti-KLK5 antibody in GNP and in solution $(\mathrm{pg} / \mathrm{mL}) .{ }^{\mathrm{c}}$ Concentration of anti-KLK5 antibody in solution $(\mu \mathrm{g} / \mathrm{mL})$. Values were expressed as Mean \pm SD $(\mathrm{n}=3)$. GNP.OH Followed a Boltzmann Sigmoidal model, while the rest of the particles and drugs followed a One Phase Exponential Association model

Results in Figure 6 show that the different particles/drug/antibody can actually inhibit KLK5 activity, although at different concentrations or following a different model. Equations, $\mathrm{IC}_{50}$ values and other inhibition parameters obtained can be seen in Supporting Information (Table S 2).

As expected, AEBSF $\cdot \mathrm{HCl}$ in solution inhibits KLK5 activity, which could be attributed to the covalent attachment of the fluorosulphonyl group from the drug to the hydroxyl group of the serine 
in the enzyme's active site, and thus being an irreversible inhibition. Interestingly, also control particles (GNP.OH and GNP.OH/COOH) display KLK5 inhibitory activity, suggesting a possible interaction between the GNP and the enzyme's active site, for example, the formation of hydrogen bonds or ionic bonds. These interactions might therefore affect the normal enzymatic activity.

GNP.OH showed a very different inhibitory behavior than the rest, following a Boltzmann Sigmoidal model, and providing much less inhibition as compared by the $\mathrm{IC}_{50}$ values $(\mathrm{P}<0.001)$. In contrast, GNP.OH/COOH and the rest of the particles or free drug, followed a One Phase Exponential Association model, reaching inhibitions higher than $80 \%$. No significant differences $(\mathrm{P}>0.05)$ were obtained between the inhibitory activity of the particles $\mathbf{G N P . O H} / \mathbf{C O O H}$, GNP.AEBSF, GNP.COOH AEBSF, GNP.antiKLK5 or the free AEBSF.HCl; however, when the drug is ionically bonded in GNP.COOH AEBSF, a slight enhancement of the inhibition can be appreciated, which could be due to the non-covalent nature of the attachment, which permits some drug release, and therefore both the drug and the nanoparticles contribute to the overall inhibition. Statistical analysis can be seen in Supporting Information (Table S 4).

Finally, the free anti-KLK5 did not show inhibitory activity at similar concentrations as when it is in GNP.antiKLK5 $(50-1200 \mathrm{pg} / \mathrm{mL})$. This can be explained as the molar concentration of KLK5 in the assay is almost one million times higher than the concentration of antibody. However, when the concentration of antibody is increased $(1-20 \mu \mathrm{g} / \mathrm{mL})$, inhibition is achieved, which also follows an exponential behavior. These results show that the interaction between the anti-KLK5 antibody and the enzyme can actually inhibit the enzymatic activity, and which, to our knowledge, was not previously reported.

\section{Internalization of GNPs in human keratinocytes}


To test the ability of gold nanoparticles to be internalized in human keratinocytes (HaCaT), GNP.OH/COOH nanoparticles were labelled with the fluorophore BODIPY by covalently bonding the fluorophore to the carboxylic acid groups forming an amide bond. Labelled particles were added to keratinocytes at a concentration of $30 \mu \mathrm{M}$ of active groups. After 24 hours of incubation at $37^{\circ} \mathrm{C}$, internalization of nanoparticles was visualized by confocal fluorescence microscopy (Figure 7). As expected, no red signal was observed in non-treated control cells. In contrast, BODIPY-labelled nanoparticles were visualized in the cytoplasm of HaCaT cells incubated at $37^{\circ} \mathrm{C}$. Remarkably, the area of the nucleus is observed completely dark, showing that the particles do not enter the cell nucleus, for which they should not induce damage to DNA.

The cell internalization of drugs or proteins is hampered by their physicochemical properties; therefore, these particles could be used as an intracellular delivery system. For example, the potential use of anti-KLK5 antibody to block KLK5 in vivo, is hampered by the inability of antibodies to enter the target cells, as it is known that the cell membrane represents a nonpermissive barrier for the transport of antibodies across it. Therefore, as it is the case of GNP.antiKLK5, these nanoparticles could be used to drive cellular uptake of antibodies and target cytosolic KLK5 in HaCaT cells. 

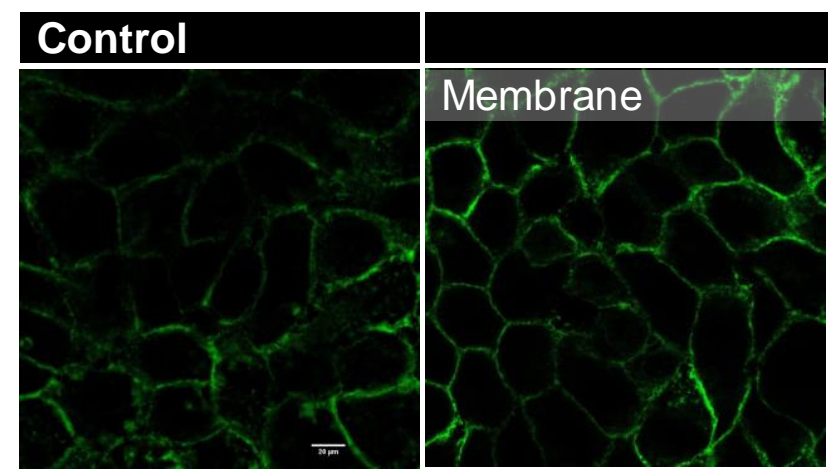

\section{HaCaT + GNP}

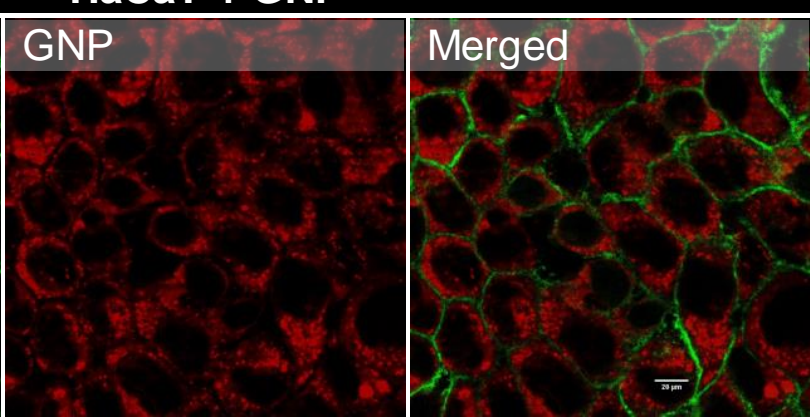

Figure 7. Internalization of BODIPY-labeled GNP.OH/COOH in HaCaT keratinocytes by confocal fluorescece microscopy. HaCaT cells were incubated for 24 hours with $30 \mu \mathrm{M}$ of GNP.OH/COOH. Images show cell membranes counterstained with wheat germ agglutiningAlexa-594 (green) and internalized BODIPY-labelled particles (red). Analysis was performed in a Leica TCS SP5 laser scanning confocal spectral microscope with 63x oil immersion objective lens, and images were captured with a Nikon color camera (16 bit). Scale bar: $20 \mu \mathrm{m}$.

\section{Cytotoxicity assays}

Although it is generally accepted that the toxicity of gold nanoparticles is low because of the low reactivity of the material, there is a growing interest in the study of their toxicity and life expentancy. It has been seen that the size of the gold nanoparticles influences the cytotoxicity, being the ones with a gold core diameter smaller than $2 \mathrm{~nm}$ the ones that present unusual reactivity in terms of oxidative stress; ${ }^{28-30}$ however, toxicity is highly dependent on the outer functionalization of the GNP, and PEG entities relieve nanotoxicity and permit the GNP escaping the opsonization process in the immune response. ${ }^{31}$ Hence, the cellular toxicity in human keratinocytes (HaCaT cells) was evaluated after incubation with the different nanoparticles by performing the MTT assay. 
Cell viability of $\mathrm{HaCaT}$ keratinocytes was evaluated both in presence or absence of the glucan related zymosan, with the aim of setting up a cellular model that mimics Rosacea conditions, and since it is known that zymosan can activate the TLR-2 receptors, whose signalling pathway is involved in Rosacea-associated overexpression of KLK5 (see Figure 1), ${ }^{6,7,9}$ The results show that zymosan shows no cytotoxicity after $24 \mathrm{~h}$ incubation. Moreover, a possible indirect influence of zymosan in cell viability after incubation with nanoparticles was evaluated by incubating $\mathrm{HaCaT}$ cells with GNP.OH/COOH, both in presence and absence of zymosan, showing also that zymosan has no influence on viability results (data not shown). For these reasons, the cell viability in human keratinocytes (HaCaT cells) was evaluated under Rosacea conditions (zymosan-stimulated) after incubation with the different nanoparticles at concentrations up to $100 \mu \mathrm{M}$ (GNP.OH, GNP.OH/COOH, GNP.COOH AEBSF, GNP.AEBSF, and GNP.antiKLK5), as well as with the free drug AEBSF· $\mathrm{HCl}$ or the anti-KLK5 antibody (Figure 7). GNP concentrations were calculated on the basis of "active groups". In GNP.OH/COOH the term "active groups" refers to the concentration of PEG.COOH molecules. Likewise, it refers to those PEG.COOH molecules that were functionalized in case of the other nanoparticles, namely GNP.COOH AEBSF, GNP.AEBSF, and GNP.antiKLK5. As GNP.OH does not have two types of thiols, concentration was adjusted to correctly represent an equivalent concentration of total thiols than in GNP.OH/COOH taking into account the proportion of PEG.OH to PEG.COOH thiols.

GNP.OH did not cause toxicity in keratinocytes since cell viability was close to $100 \%$ at all the concentrations tested (Figure 7 A). These results were consistent with the reported alleviation of GNP toxicity by PEG functionalization. However, incubation with GNP.OH/COOH resulted in a significant decrease in cell viability, around $30 \%$ at concentrations above $5 \mu \mathrm{M}$ (Figure 7 B). This effect could be attributed to the reactivity of free $\mathrm{COOH}$ groups on the surface of these 
nanoparticles, prior to the functionalization with the desired drugs. Despite the toxicity levels observed with these particles, they should not represent a problem when the administration route is intended to be dermal, as the replication of keratinocytes in the skin occurs faster than the majority of cells in the organism, and the outer layer of the skin (stratum corneum) is entirely comprised of biologically dead cells.

In contrast, the serine protease inhibitor $\mathrm{AEBSF} \cdot \mathrm{HCl}$ was highly cytotoxic in solution, resulting in less than $10 \%$ of cell viability at $75 \mu \mathrm{M}$ and undetectable cell viability levels at $100 \mu \mathrm{M}$, which completely impairs its potential therapeutic use. This high toxicity is most probably related to the reactivity of the fluorosulphonyl group, which might be interacting with other vital biomolecules of the cell. However, drug associated cytotoxicity was significantly reduced $(\mathrm{p}<0.05)$ when AEBSF is linked to the GNP through an electrostatic bond (GNP.COOH AEBSF), and especially through a covalent bond (GNP.AEBSF). (Figure 7 C). The higher cytotoxicity observed for the electrostatically-bound AEBSF nanoparticles is therefore related to the expected dissociation of the drug at the cell culture conditions (aqueous solution, $\mathrm{pH}$ 7.4). These particles thus represent a great potential as a delivery system for highly cytotoxic drugs, as they could promote internalization while reducing drug-associated toxicity.

Incubation with GNP.antiKLK5 at concentrations up to $100 \mu \mathrm{M}$ active groups $(285 \mathrm{pg} / \mathrm{mL}$ antibody), modestly decreased cell viability to $80 \%$. In contrast, incubation with the anti-KLK5 antibody did not cause any cytotoxic effect at equivalent antibody concentrations. (Figure 7 D), and up to $1000 \mathrm{pg} / \mathrm{mL}$ of antibody (data not shown). This effect could be attributed to the presence of unreacted $\mathrm{COOH}$ groups on the GNP surface; however, the cell viability values are significantly higher with the attached antibody as compared to the nanoparticles alone (GNP.OH/COOH) $(\mathrm{P}<0.05)($ Figure 7 E). 


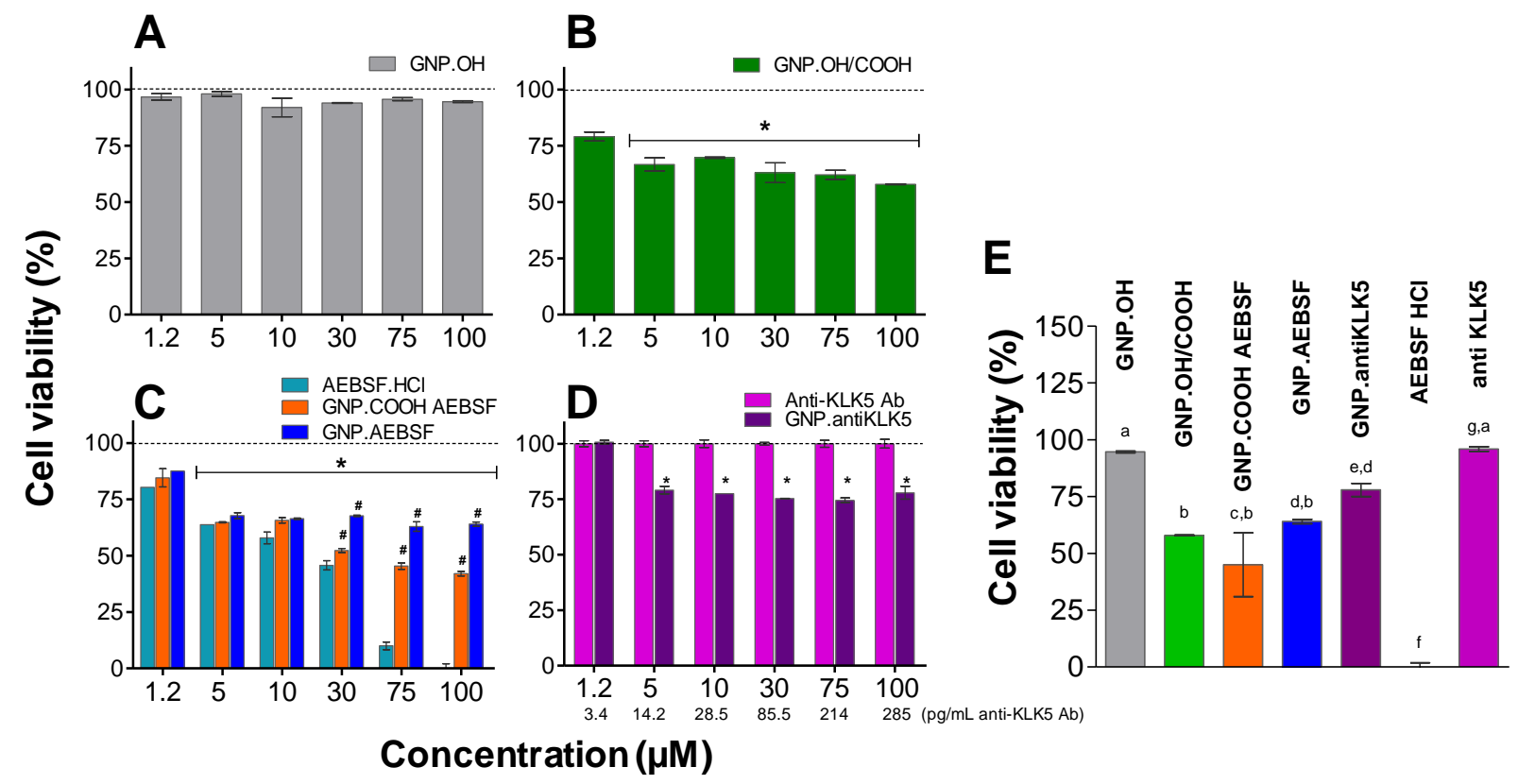

Figure 7. Cell viability of HaCaT keratinocytes after incubation for 24 hours with GNPs at different concentrations of active groups $(\mu \mathrm{M})$. Cell viability was assayed by the MTT reduction method and expressed as percentage of zymosan treated control cells (dotted line). Values represent the Mean $\pm \mathrm{SD}(\mathrm{n}=3)(* \mathrm{P}<0.05$, versus zymosan-treated control cells; \# $\mathrm{P}<0.05$, versus cells treated with AEBSF·HCl in solution). (A) GNP.OH, (B) GNP.OH/COOH, (C) GNP.COOH AEBSF and GNP.AEBSF, and (D) GNP.antiKLK5. In panels (C) and (D) free drug AEBSF.HCl and anti-KLK5 antibody are included respectively for comparison. In panel (D) the concentration of antibody $(\mathrm{pg} / \mathrm{mL})$ is also shown. (E) Comparison of cell viability among different particles/drug/antibody after $24 \mathrm{~h}$ incubation at $100 \mu \mathrm{M}$ concentration of active groups. Different letters indicate significant differences between groups $(\mathrm{P}<0.05)$. 


\section{Inhibition of the inflammatory response in human keratinocytes}

As it has been reported that TLR-2 receptor expressed in HaCaT keratinocytes can be activated by zymosan, we sought to evaluate the inflammatory response in HaCaT cells upon 24 h incubation with this glucan molecule $(100 \mu \mathrm{g} / \mathrm{mL})$, with the aim of mimicking Rosacea conditions. Incubation with zymosan simulated the inflammation observed in Rosacea patients, as cells without any stimulation showed basal IL-8 levels $(893 \pm 78 \mathrm{pg} / \mathrm{mL})$, while cells incubated with zymosan secreted 15 times more IL-8 (up to $14460 \pm 1150 \mathrm{pg} / \mathrm{mL})(\mathrm{P}<0.05)$.

Thus, the potential of the different GNP to inhibit the inflammatory response was assessed through quantification of secreted IL-8 in zymosan-stimulated HaCaT cells. Hence, keratinocytes were incubated with zymosan for $24 \mathrm{~h}$ in presence of the different GNP at different concentrations of active groups $(0.625 \mu \mathrm{M}$ to $100 \mu \mathrm{M})$. Levels of secreted IL-8 were quantified and plotted as percentage respect to the positive and negative controls versus concentration of active groups (Figure 8). 


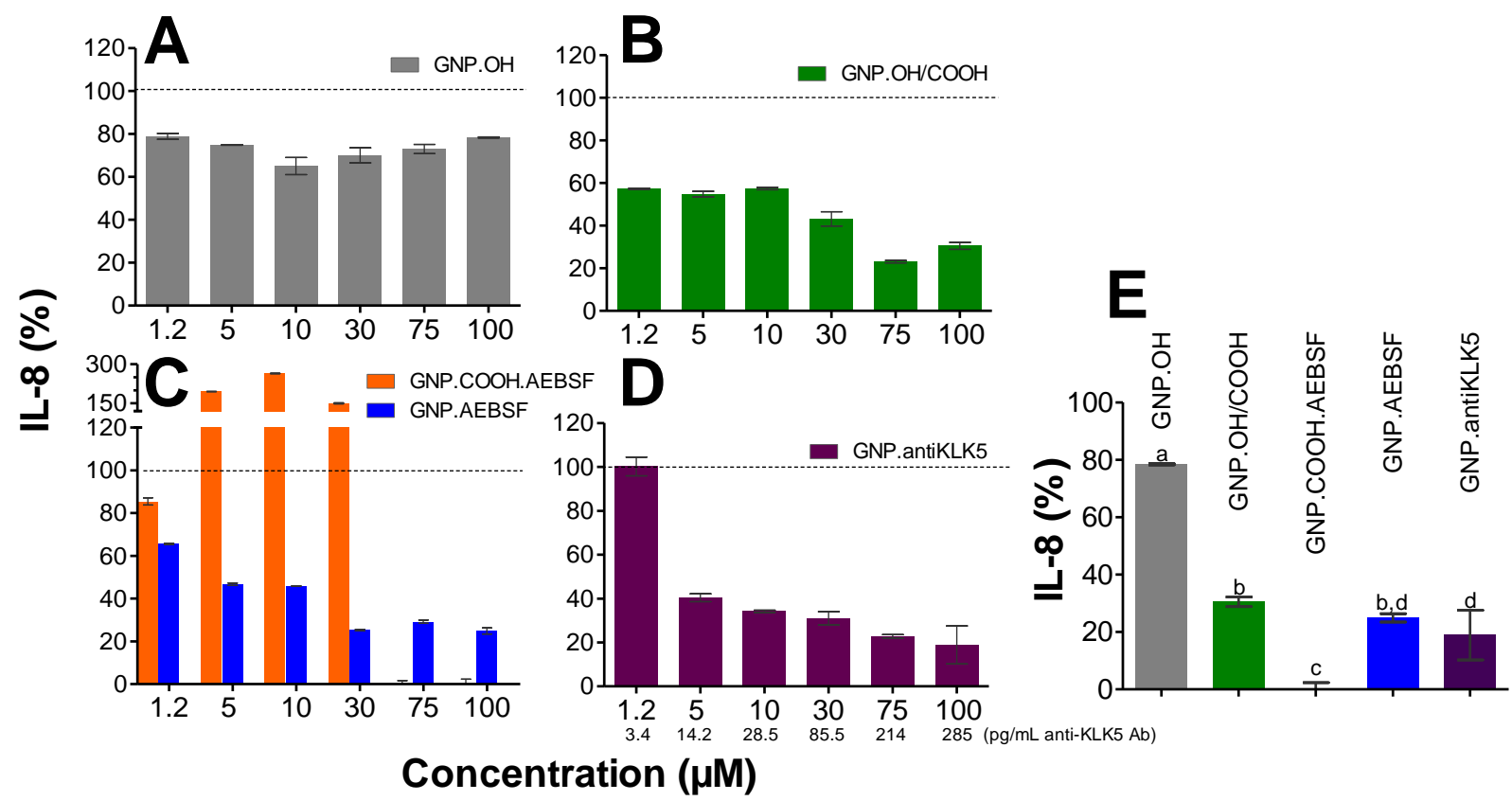

Figure 8. (A-D) IL-8 secretion in HaCaT incubated with the different nanoparticles at different concentrations of active groups. (D) Concentrations of anti-KLK5 antibody in GNP.antiKLK5 are also shown in the axis. (E) Comparison of IL-8 levels after incubation with different particles at $100 \mu \mathrm{M}$. Different letters represent significant differences $(\mathrm{P}<0.05)$. All results are plotted relative to zymosan-stimulated cells and non-stimulated cells. Values represent Mean \pm SD ( $n=3$ ).

Incubation with GNP.OH diminished only slightly, the levels of the pro-inflammatory IL-8, even at the highest concentrations tested, which is probably related to their low enzymatic inhibitory activity. In contrast, GNP.OH/COOH, GNP.AEBSF, and GNP.antiKLK5 importantly decreased IL-8 levels down to $20 \%$, even at very low concentrations. Significant differences were observed between particles at $100 \mu \mathrm{M}$, except for GNP.AEBSF and GNP.antiKLK5, which showed equivalent IL-8 levels. A completely different behavior was 
observed with GNP.COOH AEBSF, which showed an increase of IL-8 secretion at concentrations below $30 \mu \mathrm{M}$, but a decrease to undetectable levels above this concentration.

\section{Inhibition of intracellular KLK5 in human keratinocytes}

The potential of the functionalized nanoparticles to inhibit KLK5 activity ex vivo was evaluated under simulated Rosacea conditions. Hence, we measured the intracellular KLK5 activity in nonstimulated and in zymosan-stimulated keratinocytes. Also, cells were incubated with zymosan in presence of GNP.COOH AEBSF, GNP.AEBSF, GNP.antiKLK5, and GNP.OH/COOH as a control. In zymosan-stimulated keratinocytes (Control(+)), KLK5 activity was increased by $21 \%$ compared to the basal activity value in non-stimulated cells (Control(-)) (Figure 9). In this case, data did not reach statistical significance due to the wide variations in measurements. When zymosan-stimulated cells were simultaneously treated with the nanoparticles (100 $\mu \mathrm{M}$ of active groups), KLK5 activity was importantly reduced. GNP.COOH decreased the intracellular KLK5 activity reaching very similar levels to those of untreated cells (Control(-)), but particles further functionalized (GNP.COOH AEBSF, GNP.AEBSF and GNP.antiKLK5) significantly decreased activity by $35-40 \%(\mathrm{P}<0.01)$, reaching levels even below the basal value. 


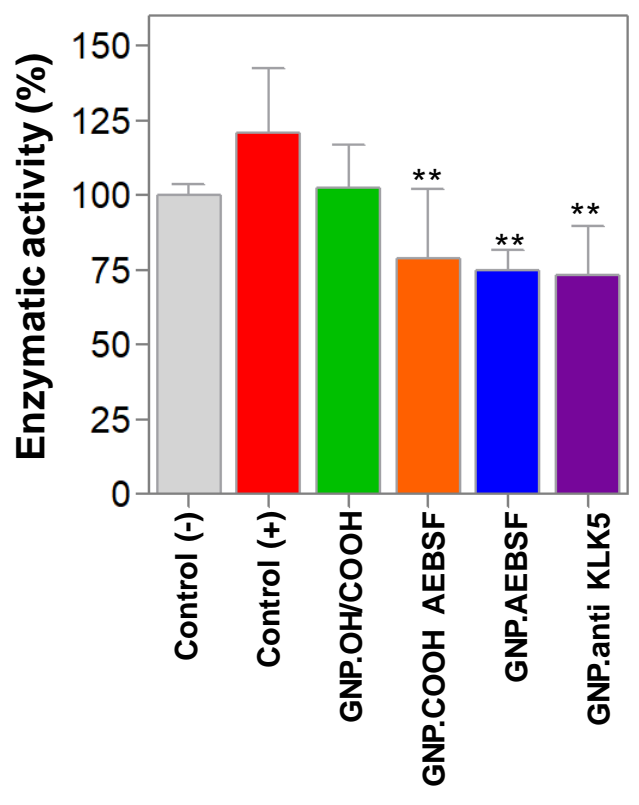

Figure 9. Intracellular KLK5 enzymatic activity of zymosan-stimulated HaCaT cells incubated with nanoparticles at $100 \mu \mathrm{M}$ active groups (GNP.COOH AEBSF (n=3), GNP.AEBSF (n=4), GNP.antiKLK5 $(n=4)$ or GNP.OH/COOH $(n=7))$. Control(-): non-stimulated cells without nanoparticles $(n=7)$. Control(+): Zymosan-stimulated cells without nanoparticles $(n=7)$. KLK5 activity is expressed as percentage of non-stimulated Control(-) cells. Values represent Mean \pm SD. **Differences with statistical significance $(\mathrm{P}<0.01)$ versus Control $(+)$.

The therapeutic potential of the different nanoparticles in inflammatory skin diseases such as Rosacea is summarized in Figure 10, showing the decrease of KLK5 activity both in vitro and intracellularly KLK5, the decrease in IL-8 secretion, and the cell viability. As it can be seen, GNP.AEBSF and GNP.anti KLK5 show high inhibitory activity of KLK5, both in vitro and intracellular, and both effectively inhibit IL-8 secretion in a similar way. According to DLS and TGA results, the number of antibodies attached to GNP.anti KLK5 should be less than that of 
AEBSF molecules attached to GNP.AEBSF. This would imply that antibody-functionalized nanoparticles have a higher efficacy than AEBSF-functionalized nanoparticles, something that could be explained because of the high specificity of the anti-KLK5 antibody towards KLK5 enzyme. Nonetheless, other unknown factors, such as the stability of the nanoparticles in the cell medium, may also influence their efficacy.

\begin{tabular}{|c|c|c|c|c|c|c|c|c|c|c|}
\hline \multirow{2}{*}{$\begin{array}{l}\text { GNP (100 } \mu \mathrm{M} \text { active groups) } \\
\text { In vitro KLK5 activity inhibition (\%) }\end{array}$} & \multicolumn{2}{|c|}{ GNP.OH } & \multicolumn{2}{|c|}{ GNP.OH/COOH } & \multicolumn{2}{|c|}{ GNP.COOH AEBSF } & \multicolumn{2}{|c|}{ GNP.AEBSF } & \multicolumn{2}{|c|}{ GNP.anti KLK5 } \\
\hline & 19 & \pm 5 & 66 & \pm 8 & 95 & \pm 1 & 72 & \pm 2 & 79 & \pm 6 \\
\hline Cell viability $(\%)^{\mathrm{a}}$ & 95 & \pm 1 & 58 & \pm 1 & 45 & \pm 14 & 64 & \pm 1 & 78 & \pm 3 \\
\hline IL-8 secretion inhibition (\%) ${ }^{\mathrm{a}}$ & 22 & \pm 1 & 70 & \pm 2 & 100 & \pm 2 & 75 & \pm 2 & 81 & \pm 9 \\
\hline Intracellular KLK5 activity inhibition (\%) ${ }^{\mathrm{a}}$ & N.A. & & 16 & \pm 14 & 35 & \pm 17 & 38 & \pm 6 & 40 & \pm 13 \\
\hline
\end{tabular}

Figure 10. Comparison of the different particles in terms of cell viability (\%), inhibition of in vitro KLK5 activity (\%), inhibition of intracellular KLK5 activity (\%) and inhibition of IL-8 secretion (\%). N.A.: Not assayed. "anhibition values respect to Zymosanstimulated HaCaT cells. Values in the bars represent the Means, values on the right of the bars represent one standard deviation.

\section{Conclusions}

Water soluble monodisperse gold nanoparticles were synthesized, comprised of a gold core ( $c a$. $1.5 \mathrm{~nm}$ diameter) and coated with a mixture of thiolated-PEG linkers of sufficient length that particles with hydrodynamic diameters around $10 \mathrm{~nm}$ (GNP.OH/COOH) are obtained, which were functionalized using the carboxyl terminal group on their surface. To explore the application of these GNP as delivery vehicles for drugs targeting key proteins altered in Rosacea, we immobilized the serine protease inhibitor $\mathrm{AEBSF} \cdot \mathrm{HCl}$ (either covalently or electrostatically) or the anti-KLK5 antibody (covalently). After functionalization, particles remain highly-water soluble giving no signs of aggregation as observed by TEM and DLS. 
GNP.OH nanoparticles did not cause toxicity in keratinocytes since cell viability was close to $100 \%$ at all the concentrations tested, but a decrease in cell viability was observed with mixed hydroxyl/carboxyl functionalized gold nanoparticles $(\mathbf{G N P . O H / C O O H})$, an effect that may be attributed to the reactivity of free $\mathrm{COOH}$ groups on the surface of these nanoparticles. However, the most remarkable observation is that although the drug AEBSF $\cdot \mathrm{HCl}$ in solution is highly cytotoxic, its incorporation to the nanoparticles through electrostatic, but especially through covalent bonding, significantly reduces its cytotoxicity, showing the high potential of the GNP to be used as drug delivery systems for highly cytotoxic drugs, as the complexation can increase drug safety. In the case of GNP.antiKLK5, the modest levels of toxicity observed are most probably related to the unreacted $\mathrm{COOH}$ groups remaining on the GNP surface. On this basis, we may speculate that a future chemical modification of these particles could lead to an increase in their safety, if after antibody attachment to the nanoparticles, the remaining $\mathrm{COOH}$ groups are blocked with other molecules, with the aim to create a non-toxic GNP surface, yet maintaining their high water solubility.

Drug- and antibody-functionalized particles, but remarkably, also GNP.OH/COOH particles without any further functionalization, inhibited in vitro KLK5 activity, in a similar way as the serine protease inhibitor $\mathrm{AEBSF} \cdot \mathrm{HCl}$ in solution.

Confocal fluorescence microscopy showed that BODIPY-labeled GNP.OH/COOH particles can be successfully internalized in $\mathrm{HaCaT}$ keratinocytes, and therefore the impairment of antibodies, other peptides, or drugs, to enter the target cells could be counteracted by their binding to these GNP.

This work provide evidence that GNP functionalized with a serine protease inhibitor either electrostatically (GNP.COOH AEBSF) or covalently (GNP.AEBSF), or with an anti-KLK5 
antibody (GNP.antiKLK5) can target cytosolic KLK5, reduce its enzymatic activity, and significantly decrease IL-8 secretion $\mathrm{HaCaT}$ cells stimulated with zymosan, therefore being a suitable delivery system in the treatment of Rosacea through this novel therapeutic strategy in the disease. Despite the efficacy of GNP.COOH AEBSF for inhibiting KLK5 both in vitro and intracellularly, as well as for decreasing the secretion of IL-8, their drug-associated cytotoxicity may compromise their future use. Instead, according to their high efficacy and safety, GNP.AEBSF and GNP.anti KLK5 are probably the most promising options for a future treatment of the disease.

Moreover, the versatility of these particles permits linking them with different drugs, antibodies or other peptides, and hence their capability for being internalized in keratinocytes, and for diminishing the toxicity of the attached drugs, makes them promising drug delivery systems in other skin diseases as well. 


\section{Experimental Section}

\section{Materials}

Chemicals: All reagents were of analytical grade. Gold(III) chloride trihydrate, tetraoctylammonium bromide, sodium borohydride, $N$-hydroxysuccinimide (NHS), $N$-(3dimethylaminopropyl)- $N$ '-ethylcarbodiimide hydrochloride (EDC), phosphate buffered saline (PBS) tablets, Trizma ${ }^{\circledR}$ base, MES monohydrate, Dulbecco's Modified Eagle's Medium (DMEM), Zymosan A from Saccharomyces cerevisiae, hydrochloric acid (reagent grade 37\%), and sodium hydroxide (pellets EMPLURA ${ }^{\circledR}$ ), and thiazolyl blue tetrazolium bromide (MTT) were purchased from Sigma-Aldrich (Germany). Sodium phosphate monobasic was purchased from Panreac (Spain). 4,4-Difluoro-5,7-dimethyl-4-bora-3a,4a-diaza-s-indacene-3-propionic acid, succinimidyl ester (BODIPY® FL) was purchased from from Setareh Biotech Eugene (USA).

Functionalized thiols HS- $\left(\mathrm{CH}_{2}\right)_{11}-\left(\mathrm{O}-\mathrm{CH}_{2}-\mathrm{CH}_{2}\right)_{6}-\mathrm{O}-\mathrm{CH}_{2}-\mathrm{COOH}$ (PEG.COOH) and HS$\left(\mathrm{CH}_{2}\right)_{11}-\left(\mathrm{O}-\mathrm{CH}_{2}-\mathrm{CH}_{2}\right)_{6}-\mathrm{OH}$ (PEG.OH) were purchased from Prochimia (Poland). 4-(2Aminoethyl)benzenesulfonyl fluoride hydrochloride $(\mathrm{AEBSF} \cdot \mathrm{HCl})$ was purchased from Fisher Scientific (USA).

Recombinant Human Kallikrein 5 and Fluorogenic Peptide Substrate BOC-Val-Pro-Arg-AMC, were purchased from R\&D systems, (USA).

4-(2-Hydroxyethyl)-1-piperazineethanesulfonic acid (HEPES), MEM Non-Essential Amino Acids Solution (100X), penicillin, streptomycin, Fetal Bovine Serum (heat inactivated), and IL-8 Human ELISA Kit, were purchased from Thermo Fisher Scientific (Life Technologies) (USA).

Solvents and buffers: Water $18 \Omega$ grade was always used, obtained from a Millipore MilliQ equipment. Dimethyl sulfoxide (DMSO) was purchased from Sigma (Germany). PBS buffer was 
prepared and used at a concentration of $10 \mathrm{mM}$ as specified by provider. MES buffer was prepared using MES monohydrate at $100 \mathrm{mM}$ concentration and adjusted to $\mathrm{pH} 5.5$ with $0.1 \mathrm{M}$ sodium hydroxide solution. Dulbecco's Modified Eagle's Medium (DMEM) was prepared as specified by provider. Enzymatic assay buffer was prepared using sodium phosphate monobasic at a concentration of $100 \mathrm{mM}$ and adjusted to $\mathrm{pH} 8$ with $0.1 \mathrm{M}$ sodium hydroxide solution. TRIS buffer was prepared using Trizma base at a concentration of $0.86 \mathrm{M}$ and adjusted to $\mathrm{pH} 7$ with $0.1 \mathrm{M}$ hydrochloric acid solution.

Filtration materials: Dialysis Membrane was a Regenerated Cellulose Tubular Membrane purchased from Cellu-Sep. Vivaspin 500 tubes with polyethersulfone membrane (100 MWCO) were purchased from Sartorius (Germany). Millex-GP syringe filters (polyether sulphone, $0.22 \mu \mathrm{m}$ pore size) were purchased from Merck Millipore (Germany).

\section{Methods}

\section{Synthesis and functionalization of gold nanoparticles}

GNP were detected in aqueous solution by the observation of a red-violet color and the presence of a characteristic SPR band near $\lambda=515 \mathrm{~nm}$ using a UV-1800 Shimadzu UV spectrophotometer (See Supporting Information, Figure S 1).

\section{Synthesis of hydroxyl gold nanoparticles (GNP.OH):}

Gold(III) chloride trihydrate $(35.5 \mathrm{mg}, 90 \mu \mathrm{mol})$ was dissolved in water $(1 \mathrm{~mL})$ and set under magnetic stirring at room temperature. A solution of tetraoctylammonium bromide (125 mg, 410 $\mu \mathrm{mol})$ in toluene $(2 \mathrm{~mL})$ was added to the gold solution. An orange emulsion was formed. Subsequently, a solution of PEG.OH $(7.3 \mathrm{mg}, 15.6 \mu \mathrm{mol})$ in water $(0.6 \mathrm{~mL})$ was added to the 
solution. Finally, a solution of sodium borohydride $(30 \mathrm{mg}, 793 \mu \mathrm{mol})$ in water $(1 \mathrm{~mL})$ was added dropwise along $5 \mathrm{~min}$ to form a violet solution during the addition. Stirring was kept for 3 hours at room temperature, and after that, GNP were partitioned with further addition of toluene $(2 \mathrm{~mL})$ and water $(2 \mathrm{~mL})$ to favor phase separation. The organic phase was extracted with water $(2 \times 2$ $\mathrm{mL}$ ). The aqueous solution of GNP was dialyzed in water for 120 hours, replacing the water in the medium once daily.

\section{Synthesis of hydroxyl-carboxyl gold nanoparticles (GNP.OH/COOH):}

Gold(III) chloride trihydrate $(67 \mathrm{mg}, 170 \mu \mathrm{mol})$ was dissolved in water $(2.5 \mathrm{~mL})$ and set under magnetic stirring at room temperature. A solution of tetraoctylammonium bromide (125 mg, 410 $\mu \mathrm{mol})$ in toluene $(5 \mathrm{~mL})$ was added to the gold solution. An orange emulsion was formed. Subsequently, a solution of PEG.OH $(6.9 \mathrm{mg}, 14.7 \mu \mathrm{mol})$ in water $(0.5 \mathrm{~mL})$ and a solution of PEG.COOH $(6 \mathrm{mg}, 11.4 \mu \mathrm{mol})$ in water $(0.5 \mathrm{~mL})$ were added to the solution in order to have a mixture of two different thiols on the surface of the GNP. Finally, a solution of sodium borohydride (33.3 $\mathrm{mg}, 880 \mu \mathrm{mol})$ in water $(2.5 \mathrm{~mL})$ was added dropwise along $5 \mathrm{~min}$ to form a violet solution during the addition. Stirring was kept for 4.5 hours at room temperature, and after that, GNP were partitioned with further addition of toluene $(2 \mathrm{~mL})$ and water $(2 \mathrm{~mL})$ to favor phase separation. The organic phase was extracted with water $(2 \times 2 \mathrm{~mL})$. The aqueous solution of GNP was dialyzed in water for 96 hours, replacing the water in the medium once daily.

\section{Ionic functionalization of gold nanoparticles with AEBSF (GNP.COOH AEBSF)}

An aliquot of GNP.OH/COOH corresponding to $0.7 \mu \mathrm{mol}$ of PEG.COOH was washed under centrifugation-resuspension cycles using Vivaspin centrifugal filter tubes $(0.5 \mathrm{~mL}$ of sample per 
tube) with enzymatic assay buffer $(2 \times 0.5 \mathrm{~mL})$. Particles were finally resuspended in $200 \mu \mathrm{L}$ of the same buffer. Afterwards, a solution of AEBSF $\cdot \mathrm{HCl}(167 \mu \mathrm{g}, 0.7 \mu \mathrm{mol})$ dissolved in assay buffer $(150 \mu \mathrm{L})$ was added to the reaction. The final concentration of both PEG.COO and AEBSF $\cdot \mathrm{HCl}$ was estimated to be $2 \mathrm{mM}$. The mixture was kept under vortex stirring for $10 \mathrm{~min}$ at room temperature in order to assure interaction of $\mathrm{AEBSF} \cdot \mathrm{HCl}$ molecules with carboxylate groups. GNP were always used freshly prepared.

\section{Covalent functionalization of gold nanoparticles with AEBSF (GNP.AEBSF)}

An aliquot of GNP.OH/COOH corresponding to $0.7 \mu \mathrm{mol}$ of PEG.COOH was washed under centrifugation-resuspension cycles using Vivaspin centrifugal filter tubes $(0.5 \mathrm{~mL}$ of sample per tube) with TRIS buffer $\mathrm{pH} 7(2 \times 0.5 \mathrm{~mL})$. Particles were resuspended in $1 \mathrm{~mL}$ of the same buffer in order to assure the $\mathrm{pH}$ of the medium for the reaction. A solution of NHS (14 $\mathrm{mg}, 130 \mu \mathrm{mol})$ and EDC ( $4 \mathrm{mg}, 20 \mu \mathrm{mol})$ in TRIS buffer $\mathrm{pH} 7(0.2 \mathrm{~mL})$ was added to the GNP and the sample was kept under vortex stirring at room temperature for 0.5 hours to activate all the carboxyl groups. Afterwards, a solution of AEBSF $\cdot \mathrm{HCl}(7 \mathrm{mg}, 29 \mu \mathrm{mol})$ in TRIS buffer $(0.2 \mathrm{~mL})$ was added to the reaction and stirring was kept for 3 hours. Particles were washed under centrifugation-redissolution cycles using Vivaspin centrifugal filter tubes $(0.5 \mathrm{~mL}$ of sample per tube) with $100 \mathrm{mM}$ MES buffer $\mathrm{pH} 5.5(4 \times 0.5 \mathrm{~mL})$ to remove non-bound AEBSF·HCl, NHS and EDC, and to adjust the $\mathrm{pH}$ to acidic conditions. Particles were resuspended in $100 \mathrm{mM}$ Phosphate $\mathrm{pH} 7$ to a final volume of $350 \mu \mathrm{L}$ just before use (final concentration of PEG.AEBSF was estimated to be $2 \mathrm{mM}$ ).

\section{Covalent functionalization of gold nanoparticles with anti-Kallikrein 5 antibody} (GNP.antiKLK5) 
An aliquot of GNP.OH/COOH corresponding to $0.7 \mu \mathrm{mol}$ of PEG.COOH was washed under centrifugation-resuspension cycles using Vivaspin centrifugal filter tubes $(0.5 \mathrm{~mL}$ of sample per tube) with TRIS buffer $\mathrm{pH} 7(2 \times 0.5 \mathrm{~mL})$. Particles were resuspended in $1 \mathrm{~mL}$ of the same buffer in order to assure the $\mathrm{pH}$ of the medium for the reaction. A solution of NHS (14 mg, $130 \mu \mathrm{mol})$ and EDC (4 mg, $20 \mu \mathrm{mol})$ in TRIS buffer pH7 $(0.2 \mathrm{~mL})$ were added to the GNP and sample was kept under vortex stirring at room temperature for 0.5 hours to activate all the carboxylic acid groups.

Afterwards, a solution of anti-Kallikrein 5 antibody $(2 \mu \mathrm{g})$ in 20 mM TRIS buffer pH 7 with 138 $\mathrm{mM} \mathrm{NaCl}(0.2 \mathrm{~mL})$, was added to the reaction and stirring was kept overnight at room temperature. Particles were washed under centrifugation-resuspension cycles using Vivaspin filter tubes $(0.5$ mL samples) with $10 \mathrm{mM}$ Phosphate buffer saline $\mathrm{pH} 7.4$ (4 x $0.5 \mathrm{~mL})$. Particles were resuspended in enzymatic assay buffer (100 mM Phosphate $\mathrm{pH}$ 7) to a final volume of $350 \mu \mathrm{L}$ (final concentration of PEG.antiKLK5 + PEG.COOH 2 mM).

\section{Water solubility and long-term stability of GNP}

The water solubility of GNP was screened by centrifuging the aqueous solution of GNP at the original concentration, using $1 \mathrm{~mL}$ Eppendorf tubes, at $16500 \mathrm{~g}$ for different times, while observing the sedimented pellet. A high-water solubility was considered when, after 30 min centrifugation at $16500 \mathrm{~g}$, a pellet of GNP could barely be observed, while leaving the supernatant of a similar color to the naked eye as before centrifugation. Aggregation of GNP was assessed by observing the occurrence of a shift to higher wavelength on the SPR band using UV-VIS spectroscopy. Spectra were recorded from freshly prepared GNP and one year after being synthesized. 


\section{Size and concentration of gold nanoparticles}

The extinction coefficient, concentration and size of GNP were estimated using UV-VIS absorption spectroscopy according to previously reported protocols. ${ }^{22}$ As the thiolated-PEG molecules are the stabilizing agents of the GNP, their complete bond to them was assumed. Therefore, the proportion of PEG.COOH to PEG.OH molecules in GNP.OH/COOH and in subsequent functionalizations was assumed to be 1:1.3. The number of thiol molecules attached per nanoparticle was estimated by dividing the calculated concentration of thiol molecules into the calculated concentration of GNP.

\section{Microscopy}

Transmission Electron Microscopy (TEM) was performed using a Tecnai SPIRIT (FEI Co.) electronic microscope at $120 \mathrm{kV}$ from the Centres Cientifics i Tècnològics de la Universitat de Barcelona (CCiTUB). Samples were analyzed by placing $5 \mu \mathrm{L}$ of nanoparticles (GNP.OH, GNP.OH/COOH, GNP.AEBSF and GNP.antiKLK5) at a concentration of $4 \mathrm{mM}$ of thiolatedPEG molecules on a carbon-coated copper grid. The images were captured by a Megaview III camera and digitalized with the iTEM program. The size of the GNP gold core was measured with the Analysis software $($ Image $\mathrm{J}()$ using the particle-count tool.

\section{LDI-TOF Mass spectrometry}

Laser desorption/ionization time-of-flight mass spectrometry (LDI-TOF-MS) analysis was performed using a 4800 Plus MALDI TOF/TOF (ABSciex - 2010) mass spectrometer, without matrix assistance, equipped with a solid state laser $(\mathrm{Nd}: \mathrm{YAG})(355 \mathrm{~nm}$, frequency $200 \mathrm{~Hz}$, pulse 
3-7 ns), and a Time of Flight analyzer in Reflective mode, at the CCiTUB. Analyses were performed in negative mode.

\section{Thermogravimetric analysis (TGA)}

Thermogravimetric analyses were performed on a Mettler-Toledo TGA-851e thermobalance at the CCiTUB. Experimental conditions: alumina crucibles of $70 \mu \mathrm{L}$ volume, atmosphere of dry nitrogen with $50 \mathrm{~mL} / \mathrm{min}$ flow rate, heating from $30^{\circ} \mathrm{C}$ to $1000{ }^{\circ} \mathrm{C}$ with a heating rate of $10^{\circ} \mathrm{C} / \mathrm{min}$. A blank curve has been previously performed and it has been subtracted.

\section{Dynamic light scattering (DLS)}

The average hydrodynamic diameter of GNP was determined through dynamic light scattering (DLS), using a Zetasizer Nano ZS, Malvern Instruments, Malvern, UK. The different GNP were diluted at different concentrations using $2 \mathrm{mM}$ PBS, and all samples were filtered using $2 \mu \mathrm{m}$ pore size syringe filters. At least three independent samples of each kind of nanoparticles were analyzed, performing 32 measurements in each analysis.

\section{In vitro enzymatic inhibition of KLK5}

Different functionalized and control GNP, previously washed with and resuspended in assay buffer $(\mathrm{pH} 8)$ to a concentration of GNP corresponding to $4.6 \mathrm{mM}$ of total thiolated-PEG molecules, were tested to inhibit KLK5 activity in solution according to the manufacturer's protocol. KLK5 was diluted to $4 \mathrm{ng} / \mu \mathrm{L}$, and fluorogenic Peptide Substrate BOC-Val-Pro-ArgAMC was diluted to $200 \mu \mathrm{M}$, both in assay buffer. $25 \mu \mathrm{L}$ of KLK5 were added to a fluorescence 
96-well plate reader. Different amounts of GNP were diluted with assay buffer to a final volume of $50 \mu \mathrm{L}$ and mixed with the KLK5 in the wells. As positive control, $50 \mu \mathrm{L}$ of assay buffer was added to plates with no GNP. Samples were incubated for 15 min under soft vortex stirring and enzymatic reaction was started by adding $50 \mu \mathrm{L}$ of $200 \mu \mathrm{M}$ fluorogenic Peptide Substrate BOCVal-Pro-Arg-AMC to wells, in which the KLK5 cleaves the bond between arginine and the fluorophore aminomethyl coumarin (AMC). Immediately, fluorescence emitted by AMC was read continuously for $10 \mathrm{~min}$ at an excitation/emission of $380 \mathrm{~nm} / 460 \mathrm{~nm}$ and plots of fluorescence against time were obtained. The slope of the positive control was taken as $100 \%$ enzymatic activity. Enzymatic activity of the samples incubated with different concentrations of GNP was calculated respect to the positive control. Enzymatic inhibition was obtained by subtracting the activity to $100 \%$ and plotted against the concentration of functional groups thought to be responsible for the inhibition ("active groups"). For GNP.OH/COOH, concentration of active groups corresponds to that of PEG.COOH groups, or the corresponding reacted group in the subsequent functionalizations (GNP.COOH AEBSF, GNP.AEBSF, GNP.antiKLK5, and GNP.COOH brimonidine). As GNP.OH only has one type of PEG.OH groups, the theoretical concentration of "active groups" was calculated to correctly represent the same concentration of total thiolated-PEG molecules by considering the proportion of the two types of thiolated-PEG molecules in GNP.OH/COOH.

Mathematical models describing the inhibition were obtained and statistical analyses were performed using Prism GraphPad software. 


\section{Cell line and growth conditions}

The human keratinocyte (HaCaT) cell line (ATCC) was routinely grown in high-glucose Dubelcco's Modified Eagle's medium (DMEM) supplemented with $25 \mathrm{mM}$ hepes, 1\% nonessential amino acids, 100U/mL penicillin, $100 \mu \mathrm{g} / \mathrm{mL}$ streptomycin, and $10 \%$ heat inactivated fetal calf serum (FCS). Cells were cultured in a humidified incubator at $37^{\circ} \mathrm{C}$ in a $5 \% \mathrm{CO}_{2}$ atmosphere and received fresh medium every 3 days. Experiments were performed at $80-90 \%$ of confluence (passes between $n=85-95)$.

\section{Internalization of GNPs in human keratinocytes}

HaCaT $\left(2 \times 10^{5}\right.$ cells $\left./ \mathrm{mL}\right)$ were seeded in 12-wells plates and grown $80-90 \%$ of confluence. GNP.OH/COOH particles were dyed with BODIPY. Briefly, an aliquot of GNP.OH/COOH corresponding to $0.7 \mu \mathrm{mol}$ of PEG.COOH was washed under centrifugation-resuspension cycles using Vivaspin centrifugal filter tubes $(0.5 \mathrm{~mL}$ of sample per tube) with TRIS buffer $\mathrm{pH} 7$ ( 2 × 0.5 $\mathrm{mL}$ ). Particles were resuspended in $1 \mathrm{~mL}$ of the same buffer in order to assure the $\mathrm{pH}$ of the medium for the reaction. Afterwards, a solution of BODIPY $(29 \mu \mathrm{mol})$ in DMSO $(0.1 \mathrm{~mL})$ was added to the reaction and stirring was kept for 3 hours. Particles were washed under centrifugationredissolution cycles using Vivaspin centrifugal filter tubes $(0.5 \mathrm{~mL}$ of sample per tube) with 100 mM Phosphate buffer pH 7 ( 4 x $0.5 \mathrm{~mL})$ to remove DMSO and non-bound BODIPY. Particles were resuspended in $100 \mathrm{mM}$ Phosphate, $\mathrm{pH} 7$ to a final volume of $350 \mu \mathrm{L}$ just before use (final concentration of total thiolated-PEG molecules was estimated to be $4.6 \mathrm{mM}$ ). BODIPY-dyed GNPs were added to cells at a concentration of $30 \mu \mathrm{M}$ of active groups. After 24-hour incubation GNP internalization was assessed by confocal fluorescence microscopy. For this, three PBS 1X washes $(3 \times 100 \mu \mathrm{L})$ steps were done to remove the non-internalized nanoparticles and the plasma 
membranes were stained for 30 minutes with $10 \mu \mathrm{g} / \mathrm{ml}$ Alexa Fluor 488-conjugated with fluorescent wheat germ agglutinin (WGA), which stains the glycocalyx membrane. Then, cells were fixed with 3\% paraformaldehyde for 30 minutes, washed with PBS 1X and covered with mounting medium (Fluoromount-G). Internalization of GNPs in HaCaT was analysed by laser scanning confocal microscopy (Leica TCS SP5), using the $63 \mathrm{X}$ oil immersion objective lens. Images were taken with a Nikon colour camera (16 bit). The fluorescent excitation wavelengths were set to $485 \mathrm{~nm}$ (red; BODIPY) and $543 \mathrm{~nm}$ (green; membrane). Z-stacks were performed with a layer interval of $0.5 \mu \mathrm{m}$. Images were analysed using Fiji image software.

\section{MTT assay}

The effect of the different GNPs on cell viability was evaluated using the MTT cytotoxicity assay (reduction of tetrazolium salt carried out by intracellular dehydrogenases of viable living cells). For this, cells $\mathrm{HaCaT}\left(2 \times 10^{5}\right.$ cells $\left./ \mathrm{mL}\right)$ were plated in 12 -wells plates for $24-48 \mathrm{~h}$ until 80 90\% of confluence and exposed to different types of nanoparticles (GNP.OH, GNP.OH/COOH, GNP.COOH AEBSF, GNP.AEBSF, and GNP.antiKLK5 as well as to AEBSF.HCl or antiKLK5 antibody in solutionin the presence of $100 \mu \mathrm{g} / \mathrm{ml}$ of zymosan. Several concentrations were tested for each type of nanoparticle (1.2-5-10-30-75-100 $\mu \mathrm{M}$ of active groups) starting from a stock solution at a concentration of $2 \mathrm{mM}$ active groups. In parallel, a positive control (incubation only with zymosan) and a negative control (cells without any stimulation) were processed for comparison. After $24 \mathrm{~h}$ incubation, cells were washed with PBS, and further incubated with fresh medium in the presence of $10 \%$ MTT for $1 \mathrm{~h}$. DMSO $99 \%$ was added to lyse the cells and dissolve the purple insoluble crystals of MTT. The cell lysate was transferred to a 96-well new plate and then the absorbance was read using a Microplate Autoreader at $\lambda=540 \mathrm{~nm}$. 


\section{Cytokine determination}

HaCaT cells were grown in 12 well plates $\left(2 \times 10^{5}\right.$ cells $\left./ \mathrm{mL}\right)$ until $80-90 \%$ confluency and exposed, to the different types of GNP (GNP.OH, GNP.OH/COOH, GNP.COOH AEBSF, GNP.AEBSF and GNP.antiKLK5) in the presence of zymosan (100 $\mu \mathrm{g} / \mathrm{ml})$. Different concentrations of GNP were tested (1.2-5-10-30-75-100 $\mu \mathrm{M}$ of active groups). HaCaT cells stimulated with zymosan without GNP were taken as the positive control, and cells without any stimulation as the negative control. Supernatants were collected after 24-hour incubation, centrifuged at $16500 \mathrm{~g}$ for 5 minutes and immediately stored at $-80{ }^{\circ} \mathrm{C}$. IL- 8 was measured using the enzyme-linked immunosorbent assay (ELISA) according to the manufacturer's manual (BD Biosciences).

\section{Intracellular KLK5 activity}

Intracellular KLK5-5 was assayed as described ${ }^{27}$ with few modifications. Briefly, HaCaT keratinocytes $\left(2 \times 10^{5}\right.$ cells $\left./ \mathrm{ml}\right)$ were grown for $24 \mathrm{~h}$ on six-well culture plates at $37^{\circ} \mathrm{C}$ in a $5 \% \mathrm{CO}_{2}$ atmosphere. Then, cells were incubated for 24 hours with GNP.OH/COOH, GNP.AEBSF and GNP.antiKLK5 at a concentration of $100 \mu \mathrm{M}$ of active groups, in the presence of zymosan (100 $\mu \mathrm{g} / \mathrm{mL}$ ). HaCaT cells stimulated with zymosan without GNP were taken as the positive control, and cells without any stimulation as the negative control. After that, cells were washed with cool $10 \mathrm{mM}$ PBS (3 X $100 \mu \mathrm{L})$, scraped and suspended in cool $10 \mathrm{mM}$ phosphate buffer $\mathrm{pH} 8(100 \mu \mathrm{L})$. Samples were lysed through sonication and centrifuged (30 min at 16,000g) to remove cells debris. Protein concentration was measured by the method of Lowry. ${ }^{32}$ The different samples were adjusted to equal protein concentration and KLK-5 activity was assayed by monitoring the 
hydrolysis of the fluorogenic Peptide Substrate BOC-Val-Pro-Arg-AMC following the protocol described by the supplier. ${ }^{27}$

\section{Statistical analysis}

In in vitro inhibition of KLK5 activity experiments, different regression models were tested using GraphPad Prism version 6.0 (GraphPad Software Inc., La Jolla, CA, United States), being the Boltzmann Sigmoidal the one that best describes inhibition provided by GNP.OH, while the One Phase Exponential Association the one that best describes inhibition in the rest of the particles or free drug.

Boltzmann Sigmoidal model is described by the equation:

$$
Y=\text { Bottom }+\frac{(\text { Top }- \text { Bottom })}{1+e^{\frac{\text { V50-X }}{\text { Slope }}}}
$$

Where $\mathrm{Y}$ is the inhibition (\%) at a certain concentration, Top is the maximum inhibition that can be achieved, Bottom is the theoretical inhibition at zero concentration, $\mathrm{X}$ represents the concentration, V50 is the concentration at which half of the maximum inhibition is reached, and slope describes the steepness of the curve.

One Phase Exponential Association model is described by the equation: $\mathrm{Y}=\mathrm{Y}_{\max }\left(1-\mathrm{e}^{-\mathrm{kX}}\right)$

Where $\mathrm{Y}$ represents the inhibition at a certain concentration, $\mathrm{Y}_{\max }$ is the maximum inhibition that can be achieved, and X represents the concentration.

Statistical analyses were performed using SPSS version 20.0 software (SPSS, Inc.) and GraphPad Prism version 6.0 (GraphPad Software Inc., La Jolla, CA, United States). Data were expressed as Mean $\pm \mathrm{SD}$, unless stated otherwise. For in vitro KLK5 inhibition, $\mathrm{IC}_{50}$ values were therefore obtained according to the corresponding equation and compared. For cytotoxicity studies, 
comparison between the different groups was performed considering the cell viabilities obtained at a concentration of $100 \mu \mathrm{M}$ of active groups. For anti-inflammatory activity of GNP, IL-8 secretion levels after incubation with nanoparticles at $100 \mu \mathrm{M}$ of active groups were compared. For the inhibition of intracellular KLK5, enzymatic activity values after incubation of cells with GNP at a concentration of $100 \mu \mathrm{M}$ of active groups were compared. Differences between more than two groups were assessed using one-way ANOVA followed by Tukey's test. Significant differences were always established at $P \leq 0.05$.

\section{ASSOCIATED CONTENT}

Supporting Information. UV-VIS spectra from GNP.OH, GNP.COOH, GNP.COOH AEBSF, GNP.AEBSF, GNP.antiKLK5, AEBSF.HCl, and antiKLK5 antibody (Figure S 1), Size distribution plots (absolute and relative counts) as observed by TEM (Figure S 2), LDI-TOF MS analyses (Figure S 3 and Figure S 4), thermogravimetric analyses (Figure S 5 -Figure S 9), Dynamic Light Scattering (Table S 1), inhibition parameters from in-vitro KLK5 inhibition (Table S 2 and Table S 3), statistical analyses from in-vitro KLK5 inhibition (Table S 4), cytotoxicity studies (Table S 5), anti-inflammatory activity of GNP (Table S 6), and inhibition of intracellular KLK5 activity (Table S 7), as well as a schematic representation of the nanoparticle functionalizations and sizes (Figure S 10), are available and can be found in the Supporting Information.

The following files are available free of charge. Supporting Information (PDF).

\section{AUTHOR INFORMATION}




\section{Corresponding Authors}

Email:mlperez@ub.edu,josefabadia@ub.edu

\section{Present Addresses}

\# School of Pharmacy, The University of Nottingham, University Park, Nottingham NG7 2RD, England, United Kingdom.

\section{Acknowlegments}

This work was supported by EU ERDF (FEDER) funds and the Spanish Government grant TEC2014-51940-C2-2-R. D.L. thanks the CONACYT for a predoctoral grant 


\section{REFERENCES}

(1) Yamasaki, K., Di Nardo, A., Bardan, A., Murakami, M., Ohtake, T., Coda, A., Dorschner, R. A., Bonnart, C., Descargues, P., Hovnanian, A., Morhenn, V. B., and Gallo, R. L. (2007) Increased serine protease activity and cathelicidin promotes skin inflammation in rosacea. Nat Med 13, 975980.

(2) Crawford, G. H., Pelle, M. T., and James, W. D. (2004) Rosacea: I. Etiology, pathogenesis, and subtype classification. J. Am. Acad. Dermatol. 51, 327-341-344.

(3) Johnson, A. W., and Johnson, S. M. (2015) The Role of Topical Brimonidine Tartrate Gel as a Novel Therapeutic Option for Persistent Facial Erythema Associated with Rosacea. Dermatol. Ther. (Heidelb). 5, 171-181.

(4) Del Rosso, J. Q. (2013) Management of facial erythema of rosacea: What is the role of topical

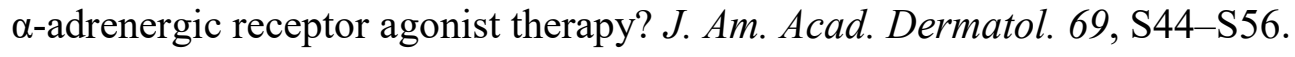

(5) Chang, B. P., Kurian, A., and Barankin, B. (2014) Rosacea: an update on medical therapies. Skin Therapy Lett. 19, 1-4.

(6) Meisgen, F., Xu Landén, N., Bouez, C., Zuccolo, M., Gueniche, A., Ståhle, M., Sonkoly, E., Breton, L., and Pivarcsi, A. (2014) Activation of Toll-like receptors alters the microRNA expression profile of keratinocytes. Exp. Dermatol. 23, 281-283.

(7) Meisgen, F., Xu Landén, N., Wang, A., Réthi, B., Bouez, C., Zuccolo, M., Gueniche, A., Ståhle, M., Sonkoly, E., Breton, L., and Pivarcsi, A. (2014) MiR-146a negatively regulates TLR2-induced inflammatory responses in keratinocytes. J. Invest. Dermatol. 134, 1931-40.

(8) Yamasaki, K., and Gallo, R. L. (2011) Rosacea as a Disease of Cathelicidins and Skin Innate 
Immunity. J. Investig. Dermatology Symp. Proc. 15, 12-15.

(9) Yamasaki, K., Kanada, K. N., Macleod, D. T., Borkowski, A. W., Morizane, S., Nakatsuji, T., Cogen, A. L., and Gallo, R. L. (2011) TLR2 Expression Is Increased in Rosacea and Stimulates Enhanced Serine Protease Production by Keratinocytes. J. Invest. Dermatol. 130, 688-697.

(10) Borgoño, C. A., Michael, I. P., Komatsu, N., Jayakumar, A., Kapadia, R., Clayman, G. L., Sotiropoulou, G., and Diamandis, E. P. (2007) A potential role for multiple tissue kallikrein serine proteases in epidermal desquamation. J. Biol. Chem. 282, 3640-3652.

(11) Fischer, J., and Meyer-Hoffert, U. (2013) Regulation of kallikrein-related peptidases in the skin - From physiology to diseases to therapeutic options. Thromb. Haemost. 110, 442-449.

(12) Michael, I. P., Sotiropoulou, G., Pampalakis, G., Magklara, A., Ghosh, M., Wasney, G., and Diamandis, E. P. (2005) Biochemical and enzymatic characterization of human kallikrein 5 (hK5), a novel serine protease potentially involved in cancer progression. J. Biol. Chem. 280, 1462814635.

(13) Two, A. M., Hata, T. R., Nakatsuji, T., Coda, A. B., Kotol, P. F., Wu, W., Shafiq, F., Huang, E. Y., and Gallo, R. L. (2014) Reduction in serine protease activity correlates with improved rosacea severity in a small, randomized pilot study of a topical serine protease inhibitor. J Invest Dermatol 13, 1143-1145.

(14) Coda, A. B., Hata, T., Miller, J., Audish, D., Kotol, P., Two, A. M., Shafiq, F., Yamasaki, K., Harper, J. C., Del Rosso, J. Q., and Gallo, R. L. (2013) Cathelicidin, kallikrein 5, and serine protease activity is inhibited during treatment of rosacea with azelaic acid $15 \%$ gel. J. Am. Acad. Dermatol. 69, 570-577. 
(15) Limón, D., Jimenez-Newman, C., Calpena, A. C., Gonzalez-Campo, A., Amabilino, D. B., and Perez-Garcia, L. (2017) Microscale coiling in bis-imidazolium supramolecular hydrogel fibres induced by release of a cationic serine protease inhibitor. Chem. Commun. 53, 4509-4512.

(16) Boisselier, E., and Astruc, D. (2009) Gold nanoparticles in nanomedicine: preparations, imaging, diagnostics, therapies and toxicity. Chem. Soc. Rev. 38, 1759-1782.

(17) Llevot, A., and Astruc, D. (2012) Applications of vectorized gold nanoparticles to the diagnosis and therapy of cancer. Chem. Soc. Rev. 41, 242-257.

(18) Ghosh, P., Han, G., De, M., Kim, C. K., and Rotello, V. M. (2008) Gold nanoparticles in delivery applications. Adv. Drug Deliv. Rev. 60, 1307-1315.

(19) Fang, J., Nakamura, H., and Maeda, H. (2011) The EPR effect: Unique features of tumor blood vessels for drug delivery, factors involved, and limitations and augmentation of the effect. Adv. Drug Deliv. Rev. 63, 136-151.

(20) Iyer, A. K., Khaled, G., Fang, J., and Maeda, H. (2006) Exploiting the enhanced permeability and retention effect for tumor targeting. Drug Discov. Today 11, 812-8.

(21) Liu, Y., Shipton, M. K., Ryan, J., Kaufman, E. D., Franzen, S., and Feldheim, D. L. (2007) Synthesis, Stability, and Cellular Internalization of Gold Nanoparticles Containing Mixed Peptides-Poly(ethylene glycol) Monolayers. Anal. Chem. 78, 2221-2229.

(22) Haiss, W., Thanh, N. T. K., Aveyard, J., and Fernig, D. G. (2007) Determination of Size and Concentration of Gold Nanoparticles from UV-Vis Spectra. Anal. Chem. 79, 4215-4221.

(23) Tiwari, P. M., Vig, K., Dennis, V. a., and Singh, S. R. (2011) Functionalized Gold Nanoparticles and Their Biomedical Applications. Nanomaterials 1, 31-63. 
(24) Eck, W., Craig, G., Sigdel, A., Ritter, G., Old, L. J., Tang, L., Brennan, M. F., Allen, P. J., and Mason, M. D. (2008) PEGylated gold nanoparticles conjugated to monoclonal F19 antibodies as targeted labeling agents for human pancreatic carcinoma tissue. ACS Nano 2, 2263-2272.

(25) Dreaden, E. C., Alkilany, A. M., Huang, X., Murphy, C. J., and El-Sayed, M. A. (2012) The golden age: gold nanoparticles for biomedicine. Chem. Soc. Rev. 41, 2740-2779.

(26) Sakabe, J. I., Yamamoto, M., Hirakawa, S., Motoyama, A., Ohta, I., Tatsuno, K., Ito, T., Kabashima, K., Hibino, T., and Tokura, Y. (2013) Kallikrein-related peptidase 5 functions in proteolytic processing of profilaggrin in cultured human keratinocytes. J. Biol. Chem. 288, 1717917189.

(27) R\&D Systems. Recombinant Human Kallikrein 5 Protein, CF 1108-SE-010: R\&D Systems.

(28) Pan, Y., Leifert, A., Ruau, D., Neuss, S., Bornemann, J., Schmid, G., Brandau, W., Simon, U., and Jahnen-Dechent, W. (2009) Gold Nanoparticles of Diameter $1.4 \mathrm{~nm}$ Trigger Necrosis by Oxidative Stress and Mitochondrial Damage. Small 5, 2067-2076.

(29) Alkilany, A. M., and Murphy, C. J. (2010) Toxicity and cellular uptake of gold nanoparticles: what we have learned so far? J. Nanopart. Res. 12, 2313-2333.

(30) Turner, M., Golovko, V. B., Vaughan, O. P. H., Abdulkin, P., Berenguer-Murcia, A., Tikhov, M. S., Johnson, B. F. G., and Lambert, R. M. (2008) Selective oxidation with dioxygen by gold nanoparticle catalysts derived from 55-atom clusters. Nature 454, 981-983.

(31) Simpson, C. A., Agrawal, A. C., Balinski, A., Harkness, K. M., Cliffel, D. E., and Al, S. E. T. (2011) Short-Chain PEG Mixed Monolayer Protected Gold Clusters Increase Clearance and Red Blood Cell Counts. ACS Nano 5, 3577-3584. 
(32) Lowry, O. H., Rosebrough, N. J., Farr, A. L., and Randall, R. J. (1951) Protein measurement with the Folin Phenol reagent. J. Biol. Chem. 193, 265-275. 


\section{Table of Contents Graphic}

\section{Multifunctional serine protease inhibitor-coated}

water-soluble gold nanoparticles as a novel targeted approach for the treatment of inflammatory skin

\section{diseases}

David Limón, ${ }^{\dagger, \ddagger}$ María José Fábrega, ${ }^{\S, l}$ Ana C. Calpena, ${ }^{\ddagger}, \mid$ Josefa Badia, ${ }^{\S, l, *}$ Laura Baldomà, and Lluïsa Pérez-Garcíał,,*\#\#

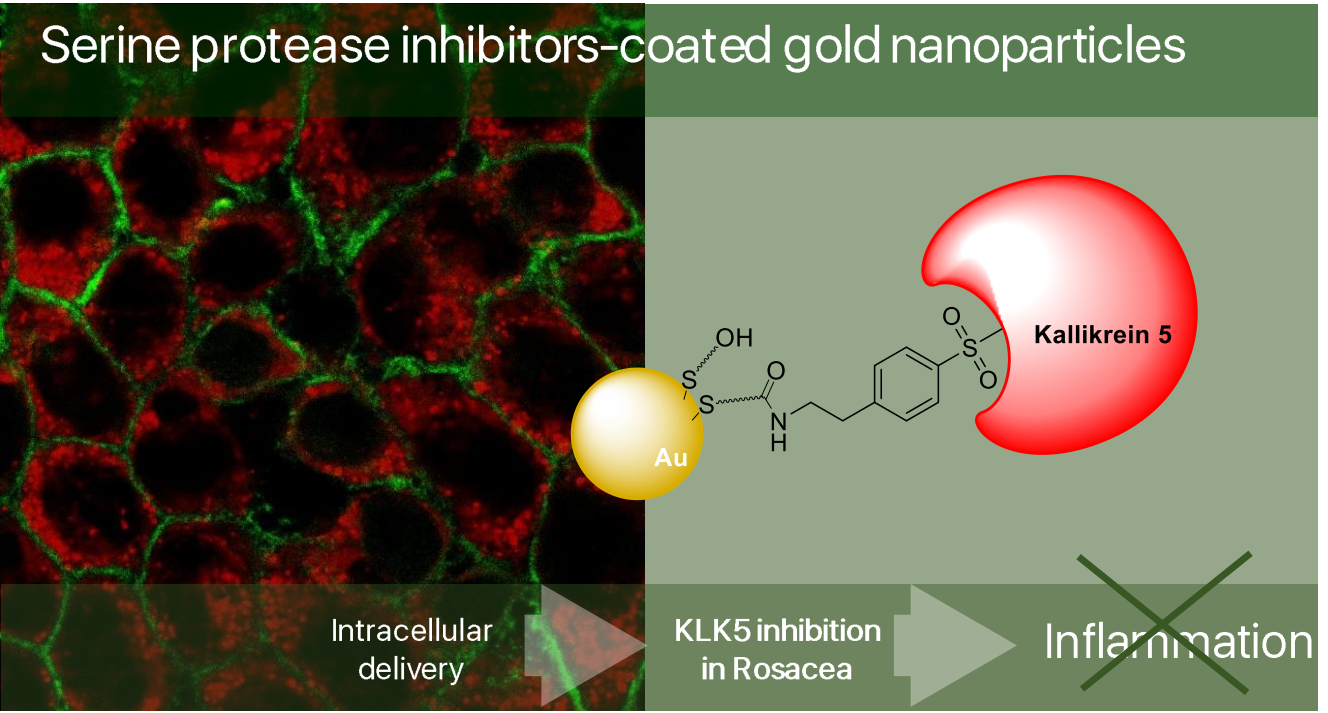

